University of Wollongong

Research Online

2013

\title{
Law of effect models and choice between many alternatives
}

Michael Alexander Navakatikyan

University of Wollongong, mnavakat@uow.edu.au

Paul Murrell

University of Auckland

Joshua Bensemann

University of Auckland

Michael Davison

University of Auckland

Douglas Elliffe

University of Auckland

Follow this and additional works at: https://ro.uow.edu.au/ahsri

Research Online is the open access institutional repository for the University of Wollongong. For further information contact the UOW Library: research-pubs@uow.edu.au 


\title{
Law of effect models and choice between many alternatives
}

\begin{abstract}
Data from five experiments on choice between more than two variable-interval schedules were modeled with different equations for the Law of Effect. Navakatikyan's (2007) component-functions models with three, four and five free parameters were compared with Stevens' (1957), Herrnstein's (1970) and Davison and Hunter's (1976) equations. These latter models are consistent with the generalized-matching principle, whereas Navakatikyan's models are not. Navakatikyan's models performed better or on par with their competitors, especially in predicting residence-time data and generalized-matching sensitivities for time allocation. The models described well an observed decrease, in several of these data sets, in generalized-matching sensitivity between two alternatives when reinforcer rate increased on the other alternatives. Models built on the generalized-matching principle cannot do this. Navakatikyan's models also performed better, though to a lesser extent, than their competitors for data sets that are not obviously inconsistent with generalized matching.
\end{abstract}

\section{Keywords}

many, between, choice, models, law, alternatives, effect

\section{Publication Details}

M. Alexander. Navakatikyan, P. Murrell, J. Bensemann, M. Davison \& D. Elliffe, "Law of effect models and choice between many alternatives", Journal of the Experimental Analysis of Behavior 1002 (2013)

222-256. 
LAW OF EFFECT MODELS AND CHOICE BETWEEN MANY ALTERNATIVES

Date of submission: Nov 26, 2012 


\section{Abstract}

Data from 5 experiments on choice between more than 2 variable-interval schedules were modeled with different equations for the Law of Effect. Navakatikyan's (2007) component-functions models with 3, 4 and 5 free parameters were compared with Stevens' (1957), Herrnstein's (1970) and Davison and Hunter's (1976) equations. These latter models are consistent with the generalized-matching principle, whereas Navakatikyan's models are not. Navakatikyan's models performed better or on par with their competitors, especially in predicting residence-time data and generalized-matching sensitivities for time allocation. The models described well an observed decrease, in several of these data sets, in generalizedmatching sensitivity between two alternatives when reinforcer rate increases on the other alternatives. Models built on the generalized-matching principle cannot do this. Navakatikyan's models also performed better, though to a lesser extent, than their competitors for data sets that are not obviously inconsistent with generalized matching.

Keywords: multi-alternative choice, mathematical models, VI schedules

Running head: Multi-alternative models for the law of effect 
Behavior in life is often related to the choice between the two most important alternatives. Nevertheless, other alternatives are often present and a more complicated choice has to be made. The existing quantitative laws of effect have been tested almost exclusively on data from choice between two alternatives. The purpose of this paper is to compare competing steady-state equations of the Law of Effect (LOE) as descriptions of data from experiments with choice between more than two alternatives.

A general LOE equation is a description of behavior, for example response rate (B), as a function of reinforcer rate $(\mathrm{R})$, or $\mathrm{B}=\mathrm{f}(\mathrm{R})$. Steady-state behavior is behavior in equilibrium, or stable behavior. LOE equations are molar, because they are concerned primarily with describing behavior and reinforcers in the steady state and aggregated over relatively long times, usually several training sessions, although they have been applied to data collected over much shorter periods.

Practically all studies of behavior in choice situations have been primarily concerned with the description of relative choice as a function of relative reinforcer rate, rather than the absolute levels of behavior. Choice in these experiments is usually well described by a power function between response- and reinforcer-rate ratios (Baum, 1974; Lander \& Irwin, 1968; Staddon, 1968; White \& Davison, 1973). This has become known as the generalized matching law (GML: Baum, 1974):

$$
\frac{B_{1}}{B_{2}}=c \cdot\left(\frac{R_{1}}{R_{2}}\right)^{a}
$$

which is linear when transformed logarithmically:

$$
\log \left(\frac{B_{1}}{B_{2}}\right)=a \cdot \log \left(\frac{R_{1}}{R_{2}}\right)+\log c .
$$

In Equations 1 and 2, $B$ measures responses emitted or time spent, and $R$ measures reinforcers obtained, at the alternatives denoted by the subscripts. Equation 2 is a straight line defined by its slope and intercept. The slope $a$ is called sensitivity to reinforcement or, more strictly, sensitivity to reinforcer-rate ratio (Lander \& Irwin, 1968; Lobb \& Davison, 1975) and describes the extent to which changes in the reinforcer ratio produce changes in the response ratio. The intercept $\log c$ is called bias (Baum, 1974) and measures any systematic preference between the alternatives not accounted for by variation in the reinforcer ratio.

The GML can be derived by transformation of various molar LOE equations, such as those suggested by Davison and Hunter (1976), and Stevens (1957). These will be described in the following sections.

The GML and these LOE equations are consistent with the principle of independence from irrelevant alternatives, or the constant-ratio rule (Luce, 1959, 1977). This principle states that choice, as measured by the ratio 
of behavior between a pair of alternatives with reinforcer rates held constant, should be independent of the existence, or value, of other alternatives, and is a consequence of the choice axiom (Luce, 1959). However, the GML is additionally an embodiment of an even stronger independence assumption, that of matching (Allen, 1981, 1982) or relative homogeneity (Prelec, 1984). According to the matching (or more accurately, generalized matching) principle, choice is unaffected by proportional increase or decrease in reinforcer rates on alternatives, and the unique function describing this principle is a power function, the GML (Allen). By default, generalized matching implies independence from irrelevant alternatives (or the relative independence assumption in Prelec's derivation).

To check if the assumption of generalized matching is violated, it is common to estimate the constancy of sensitivity parameter $a$, or equivalently, to check if the choice data in log-log coordinates are well described by a straight line (Equation 2). There are several experiments where it is shown that this assumption is violated (Alsop \& Elliffe, 1988; Elliffe \& Alsop, 1996; Elliffe \& Davison, 2010; Boelens, Kop, Nagel, \& Slangen, 1987; Davison \& McCarthy, 1994; Murrell, 1995). In particular, systematic changes in sensitivity with increase of overall reinforcer rate in concurrent variable-interval (VI) VI schedules were reported by Alsop and Elliffe (1988) and Elliffe and Alsop (1996).

Navakatikyan (2007) proposed a component-functions model of choice behavior that is not based on the generalized matching principle, and does not assume the constancy of sensitivity parameters. He successfully used this model to predict the residence time data of Alsop and Elliffe (1988) and Elliffe and Alsop (1996). (Residence time is the time spent on each alternative between switches.) Navakatikyan's predictions compared favorably against molar LOE models proposed by Davison and Hunter (1976), Herrnstein (1970), Stevens (1957) and McDowell (1986). As a by-product of the successful prediction of changes in residence time, the changes in the sensitivity parameter were well described. According to Navakatikyan, sensitivity is predicted to change when the overall reinforcer rate changes (Fig.1A), as well as when overall rates are kept constant but the ratio $R_{1} / R_{2}$, and hence relative rate, changes (Fig. 1B).

\section{PLEASE INSERT FIGURE 1 ABOUT HERE}

Navakatikyan and Davison (2010) successfully extended the component-functions model to dynamical data. They showed that the model's predictions adequately fitted data produced by negative-slope feedback functions 
(Vaughan \& Miller, 1985), concurrent variable-ratio (VR) schedules (Herrnstein \& Loveland, 1975; Mazur, 1992;

Mazur \& Ratti, 1991), Vaughan's (1981) melioration experiment, and experiments with arranged equal (Herrnstein \& Vaughan, 1980) and constant-ratio unequal (Horner \& Staddon, 1987) local reinforcer rates. Again, the model's predictions compared favorably against those of Davison and Hunter (1976) and Herrnstein (1970).

In the present article, we continue to explore the component-functions models by applying them to existing data from experiments with more than two alternatives. The preliminary analysis, using models fitted in Navakatikyan (2007), shows that if a third alternative is introduced into the model, sensitivity to the constant $R_{1} / R_{2}$ ratio will decrease with an increase in relative reinforcer rate on the third alternative if overall reinforcer rate is kept constant (Fig. 1C). We compare the performance of Navakatikyan's models with the molar models of Herrnstein (1970), Davison and Hunter (1976) and Stevens (1957) that are consistent with the generalized matching principle. Superiority of Navakatikyan's models would indicate the breach of the generalized matching principle.

Thus the primary goal of this article is to assess if Navakatikyan's (2007) models have advantages in describing multi-alternative choice, and in particular how well they might describe violation of the generalizedmatching principle, as reported in the papers cited earlier.

Five experiments with multi-alternative concurrent VI schedules were chosen: (a) two experiments with three-alternative interdependent schedules (Davison \& McCarthy, 1994; Murrell, 1995); (b) two experiments with three-alternative independent schedules (Davison \& Hunter, 1976; Pliskoff and Brown, 1976); and (c) a fouralternative dynamical (rapid-change-of-procedure) experiment (Elliffe \& Davison, 2010). Of these experiments, Davison and Hunter (1976) and Pliskoff and Brown (1976) results were reported as consistent with assumption of the GML, the other results were not.

The data sets were selected based on several criteria. The primary data needed to be readily available; excluding, for example, Jensen and Neuringer's (2009) five-alternative experiment. Also, experiments that did not use the same type of schedule, say VI schedules, for all alternatives, such as Davison's (1982) experiment, were excluded as presenting an additional complication to the multi-alternative description. Chain-schedule procedures were excluded for the same reason. Miller and Loveland's (1974) five-alternative experiment contains only five data points per bird: this was deemed insufficient and was also excluded.

In this introduction, we will describe (a) competing steady-state LOE equations; and (b) data analysis.

\section{Equations for the Law of Effect}


Residence time, response rate and changeover rate data were modeled using molar LOE equations: Stevens' (1957), Herrnstein's (1970), Davison and Hunter's (1976), and a range of Navakatikyan's (2007) equations.

\section{Stevens' (1957) LOE Model: No Interaction Between Alternatives}

Baum $(1974,1979)$ pointed out that generalized matching might be related to Stevens's (1957)

psychophysical law, which is a power function. Using behavioral notation the LOE model for an alternative can be written as Equation 3:

$$
B_{1}=A R_{1}^{d}
$$

where $d$ and $A$ are constants, $B$ is behavior, i.e., responses per min, or residence time; $R$ is the absolute rate of reinforcers per min. The constant $d$ can be interpreted as a sensitivity parameter, $a$ from Equations 1 and $2-$ the larger $d$, the faster response rate increases with increases in reinforcer rate. Equation 3 has certain limitations as an LOE model: (a) it does not have an upper bound, which should exist for response and changeover rate; and (b) it does not allow for behavioral contrast - response for each alternative is independent from responses for other alternatives, because the response rate is calculated only from its own reinforcer rate (Equation 3).

Such a simple model under normal circumstances would not be a good model of choice, and that was precisely the reason for its inclusion in the list. Some experiments are bound by their design to exhibit simple behavior and to be described well by a simple model - for example, if reinforcer rates on different alternatives were not arranged to vary independently. In this case, a more complex model (i.e., with fewer parameters) will be at a disadvantage during the selection process.

\section{Herrnstein's (1970) and Davison and Hunter's (1976) LOE Models}

Herrnstein's (1970) and Davison and Hunter's (1976) equations describe how the total amount of behavior (Herrnstein, 1974) is distributed according to the proportion of obtained reinforcers. Herrnstein's equation implies the matching principle, i.e., the value of sensitivity $a=1$ in Equation 2:

$$
B_{1}=B_{\text {max }} \frac{R_{1}}{\left(\sum_{i=1}^{n} R_{i}\right)+k},
$$

where $B_{\max }$ is a constant, representing "the total amount of behavior generated by all the reinforcements operating on the subject at a given time" (Herrnstein, 1974; p.161), or the maximum overall response rate; $k$ is a constant that is interpreted here as a general free parameter (Herrnstein's $R_{0}$ ), originally representing the unknown aggregated reinforcers for responses unaccounted for in the summation in the denominator; and $i$ is an index that covers all 
alternative responses measured in the situation. The smaller the constant $k$, the faster the response rate changes, opposite to the effect of the constant $k$ from Equation 3.

Davison and Hunter's (1976) equation implies the generalized matching principle, i.e., it has a power function in the denominator:

$$
B_{1}=B_{\max } \frac{R_{1}^{a}}{\sum_{1}^{n} R_{i}^{a}+k},
$$

where $a$ is a sensitivity parameter. Both Herrnstein's (1970) and Davison and Hunter's (1976) LOE equations allow for behavioral contrast.

Using analogy with Michaelis-Menten kinetics of enzyme-substrate reaction (e.g., Ainsworth, 1977), the mechanism for behavioral contrast in Equations 4 and 5 was classified as competitive inhibition by other reinforcers by Navakatikyan and Davison (2010). In short, this mechanism implies that reinforcers compete with each other for responses that are limited by $B_{\max }$, according to a linear (Herrnstein, 1970) or a power (Davison \& Hunter, 1976) function of their values (see Navakatikyan \& Davison for detail).

The models can be similarly described in their relation to the choice axiom. Stevens', Herrnstein's, and Davison and Hunter's LOE equations are consistent with the choice axiom, while both Stevens' and Davison and Hunter's models are consistent with the generalized matching principle.

Another of Davison and Hunter's (1976) models $\left(B_{1}=B_{\max }\left[R_{1} /\left(R_{1}+R_{\text {oth }}+k\right)\right]^{s}\right)$, was assessed, but it performed worse or on par with Equation 5 and is not presented here (see Table A1, Appendix A for the full set of models consistent with the matching principle).

\section{Navakatikyan's (2007) LOE Models}

Unlike Herrnstein's (1970) and Davison and Hunter's (1976) LOE equations, Navakatikyan's (2007) models' mechanism for behavioral contrast can be classified as non-competitive inhibition (Navakatikyan \& Davison, 2010). Navakatikyan hypothesized that, for a single-response schedule, reinforcers affect responding in a way similar to Herrnstein's (1970) LOE equation, e.g., if we assume a hyperbolic function:

$$
B=B_{\max } R /(R+k),
$$

However, when other reinforcers are present, they decrease the maximally achievable response rate. This is achieved by multiplication of Equation 6 by some decreasing function of reinforcers from other alternatives.

More generally, Navakatikyan's (2007) LOE model is a product of two functions, one increasing and one decreasing, termed component-functions. The functions are distinguished by the reinforcers that are arguments of 
these functions, termed enhancing and reducing functions, meaning that they respectively enhance or reduce a particular behavior (the latter providing the mechanism for behavioral contrast). Accordingly, functions of the first category of reinforcers are termed enhancing-component functions, and functions of the second category of reinforcers are termed reducing-component functions. Behavior is a product of the component functions plus a constant:

$$
B_{1}=F_{e n h}\left(R_{1}\right) \cdot F_{r e d}\left(\sum R_{o t h}\right)+B_{a}
$$

where $F_{\text {enh }}$ and $F_{\text {red }}$ are the enhancing- and reducing-component functions of enhancing and reducing reinforcers, respectively, and $R_{1}$ is reinforcer rate on current alternative - enhancing reinforcer rate; $\sum R_{\mathrm{oth}}$ is a sum of all reinforcers for other alternatives - reducing reinforcers; and $B_{\mathrm{a}}$ is a baseline constant.

The principle, embodied in Equation 7 can be regarded as a multiplicative interaction between current and other choices. In words, it can be formulated as follows: if the rate of obtaining one of the reinforcers changes, the resulting change in behavior occurs in proportion to the change of its component function, and also in proportion to the other component function (see Navakatikyan, 2007, for more detail). This formulation can be written as a pair of partial differential equations:

$$
\begin{aligned}
& \frac{\partial B_{1}}{\partial R_{1}}=\frac{\partial F_{e n h}}{\partial R_{1}} \cdot F_{r e d}, \\
& \frac{\partial B_{1}}{\partial R_{\text {oth }}}=\frac{\partial F_{r e d}}{\partial R_{\text {oth }}} \cdot F_{e n h} .
\end{aligned}
$$

The solution of these differential equations is Equation 7.

The only requirement for the component functions of Equation 7 is that one of them has to be increasing and one of them has to be decreasing. We did not assume that the behavior described by Equation 7 is optimal in terms of maximizing obtained reinforcer rate. However, we reasoned that such behavior should be adaptive; an organism can produce any response function that it deemed to be adaptive. One of the simplest responses would be to allocate more behavior to the current alternative in response to an increase in current reinforcer rate; and to allocate less behavior to the current alternative in response to increases in other reinforcer rates. We postulated that the responses to the current and other alternatives interact multiplicatively, while the actual shape of the component functions related to the current and other alternatives are uncertain at this stage, but can be influenced by an environment, i.e. reinforcement schedule, and by the physiology of an organism. 
The baseline constant arises in the model as a solution to the differential equations mentioned above. There are several possible interpretations of this constant. It may be that exploratory behavior provides some baseline level of activity. It may be affected by peculiarities of the reinforcer schedule, such as changeover delay, that add some constant time interval to residence time. It could be that under conditions of restricted stimulus discrimination some percentage of responses is allocated randomly. The baseline constant can be zero in some situations, as in Navakatikyan and Davison (2010).

In the analysis of residence time in concurrent VI VI schedules (Navakatikyan, 2007) a variety of functions were assessed. The best model was a product of an increasing power function with 3 parameters $\left(a+b R^{k}\right)$ and a decreasing hyperbolic function with 1 parameter $\left.\left(1 / 1+R_{\text {oth }} / k_{\text {red }}\right]\right)$ plus a baseline constant and a bias.

A similar picture emerged while applying Navakatikyan's (2007) model to dynamical data (Navakatikyan \& Davison, 2010). Three models were considered: hyperbolic, exponential and power functions for enhancing component function multiplied by a simple one-parameter decreasing hyperbola and no baseline constant. None of the models outperformed the others consistently, but all were better or on par with their competitors, thus a hyperbolic-hyperbolic model without baseline constant $\left(B=a R /(R+k) \times\left[1 /\left(1+R_{\text {oth }} / k_{\text {red }}\right]\right)\right.$ was chosen to represent the results.

At this stage we don't have a theoretical basis to choose one type of function over another in a choice situation, except for a general consideration that for the enhancing-component function unbounded functions such as exponential or power might be preferable in describing residence time, which is not naturally limited; while bounded functions, such as hyperbolae, might be better in describing response rate, which has a natural ceiling. No reasoning has emerged so far as to what might define reducing-component functions. At the same time we believe that models for different behavior indices (e.g., response rate and residence time) and in different schedules can be different. It has been shown, for example, that for a single-response schedule a basic function - the hyperbola (Equation 6) is transformed into different functions through different coupling coefficients (Killeen, 1994).

Thus, we decided to assess a range of component functions for the current study because we didn't know $a$ priori which one would be the best. As enhancing-component functions we tested hyperbolic, exponential and power functions with and without an additive constant as well as a logistic and exponential-power function. As reducing-component functions we tested a hyperbolic function, a hyperbolic-power function, exponential-power function and a decreasing logistic function. Considering the choice of functions we aimed at progressively more complicated component functions and to preserve the continuity of research we included the models that were 
explored in Navakatikyan and Davison (2010) and the best models from Navakatikyan (2007). The functions, as well as the list of all 20 models are presented in Appendix A (Table A2 \& A3).

However, again, no single model appeared to be the best to describe data in all data sets, though almost all of them displayed superior or on par performance with the models based on the matching principle. Out of 10 subsets of data that we used over 5 different experiments, only in two subsets did the models based on matching principle perform as well as Navakatikyan's (2007) models. In another subset only two of the simplest component-function models without baseline constants (Models 1 and 3 in Appendix A, Table A3) performed worse than the competing models. In the 7 remaining subsets of the data all 20 component-functions models performed better than any of competing models.

To represent the main results of the modeling we selected six component-function models that are the closest to the competing models based on the matching principle, and that would represent the cases when componentfunctions models performed worse or on par with the competing models. We chose models with unbounded power (same as Stevens' model, Equation ,3 and close to Davison and Hunter's model, Equation 5) and bounded hyperbolic (same as Herrnstein's model, Equation 6) enhancing-component functions; and with one-parameter hyperbolic and more flexible two-parameter hyperbolic-power functions described below. Two out of six models did not have the baseline constant $B_{\mathrm{a}}$, and one of those two showed worse performance than competitors. The full list of the four competing models is in Table A3, Appendix A.

While naming the models we will use the name of the enhancing-component function first and separate the component-functions by the product symbol $(\times)$. Thus the following LOE models, as written for the first alternative, were presented:

Hyperbolicxhyperbolic models without and with baseline constant (hypxhyp, hyp $\times h y p+b)$ :

$$
\begin{aligned}
& B_{1}=B_{\max } \frac{R_{1}}{R_{1}+k} \cdot \frac{1}{1+\left(\sum R_{\text {oth }}\right) / k_{\text {red }}}, \\
& B_{1}=B_{\max } \frac{R_{1}}{R_{1}+k} \cdot \frac{1}{1+\left(\sum R_{\text {oth }}\right) / k_{\text {red }}}+B_{a} .
\end{aligned}
$$

Power×hyperbolic models without and with baseline constant (pow $\times$ hyp, pow $\times h y p+b)$ :

$$
\begin{aligned}
& B_{1}=A R_{1}^{d} \cdot \frac{1}{1+\left(\sum R_{\text {oth }}\right) / k_{\text {red }}}, \\
& B_{1}=A R_{1}^{d} \cdot \frac{1}{1+\left(\sum R_{\text {oth }}\right) / k_{\text {red }}}+B_{a} .
\end{aligned}
$$


Hyperbolicxhyperbolic-power and power×hyperbolic-power models with baseline constant (hyp $\times$ hypp $+b$, pow $\times h y p p+b)$ :

$$
\begin{aligned}
& B_{1}=B_{\max } \frac{R_{1}}{R_{1}+k} \cdot \frac{1}{1+\left(\left(\sum R_{\text {oth }}\right) / k_{\text {red }}\right)^{s}}+B_{a} . \\
& B_{1}=A R_{1}^{d} \cdot \frac{1}{1+\left(\left(\sum R_{\text {oth }}\right) / k_{\text {red }}\right)^{s}}+B_{a} .
\end{aligned}
$$

where $k_{\text {red }}$, and $s$ are constants controlling how fast response rate decreases with increase of reinforcer rate on other alternatives. The smaller $k_{\text {red }}$ the faster the change; $s<1$ slows the rate and $s>1$ accelerates the rate. Note, that the expression for the reducing hyperbolic function $1 /\left(1+\left(\sum R_{\text {oth }}\right) / k_{\text {red }}\right)$ (Equations 8 to 11$)$ is equivalent to $k_{\text {red }}\left(k_{\text {red }}+\sum R_{\text {oth }}\right)$ used previously (Navakatikyan \& Davison, 2010), and introduced here to demonstrate the similarity with the hyperbolic-power function, $\left.1 /\left(1+\left(\sum R_{\mathrm{oth}}\right) / k_{\mathrm{red}}\right)^{\mathrm{s}}\right)$ in Equations 12 and 13.

\section{Reinforcer Biases}

All LOE models were fitted in two modifications - with and without reinforcer biases. The bias coefficients were assigned to each reinforcer rate except for the last one, for which the bias was kept $=1$. Thus for threealternative choice there were two biases $c_{1}$ and $c_{2}$, and the following biases (or weighted) reinforcer rates were used: $c_{1} R_{1}, c_{2} R_{2}$, and $R_{3}$. Fortunately, practically all models without biases were better according to our selection criterion, AICc (see the next section); and therefore omitted from the results.

\section{Data Analysis}

Five data sets obtained in the experiments listed above were chosen for the present analyses. There were five subsets of data on response rate, four subsets on residence time, and one subset on changeover rate. All data sets except one (Elliffe \& Davison, 2010) contain data on steady-state behavior. Models for these data sets are LOE equations and were fitted in the usual fashion. To model Elliffe and Davison's (2010) data we used the linearization technique described in Navakatikyan and Davison (2010) in detail. All models in their dynamical form have an additional dynamic constant $k_{t}$, describing the rate of behavioral change. A brief description of the modeling approach is in Appendix B.

The details of the statistical analysis are placed in Appendix C. In brief, as a goodness of fit measure we used variance accounted for, $\mathrm{VAC}=100(\mathrm{Var}-\mathrm{RSS} / \mathrm{n}) / \mathrm{Var}$, where Var, RSS, and $\mathrm{n}$ are the variance of the behavioral index, the residual sum of squares of the fitted model and the number of data points. Unless otherwise stated we present a median value of VAC across fits to individual subjects' data. 
As the models have different numbers of adjustable parameters, to select the best model we used secondorder (or corrected) Akaike information criterion (AICc) (e.g., Burnham \& Anderson, 1998). Conventionally, models are compared using the differences ( $\triangle \mathrm{AICc}$ ) between values of a model's AICc and the AICc of the best model. A conservative cutoff value of 10 for $\triangle \mathrm{AICc}$ was chosen as a precaution against spurious conclusions. Models whose values of $\triangle \mathrm{AICc}$ are within this cutoff value are said to be a confidence set of models, i.e. they are statistically indistinguishable. To aggregate the results over all experiments the models were ordered according to the sums of $\triangle \mathrm{AICc}$ over the experiments. Three types of transformations were applied to behavioral indices to ensure the best homogeneity of variance and normality of data distribution, $\log (y), \log (y+1)$ and no transformation. The details of the data analysis are in Appendix C.

\section{Reference Conventions and the Location of Results}

The expanded and abbreviated designations of the models will be used interchangeably, for clarity. We will also omit the year of publication citing Herrnstein's (1970), Davison and Hunter's (1976), and Navakatikyan's (2007) LOE models in most cases. The best model in each experiment was one of Navakatikyan's models; accordingly, such models were presented in Figures 2 to 12. Median values of the best models' parameters for all experiments are reported in Table D1, Appendix D. Performance of all selected models for all experiments is summarized in Table 1 as $\triangle \mathrm{AICc}$, VAC for behavioral index and GML sensitivity, and a flag to signify that the test for trend in residuals was passed.

\section{Modeling 1: Interdependent Concurrent VI VI VI Schedules, Steady State Data}

The first two data sets were taken from Davison and McCarthy (1994) and from Murrell (1995). Both experiments had identical random-switching procedures, but differently arranged reinforcer rates - in more deliberate fashion in the latter experiment. Reinforcers were dependently scheduled in both experiments. Both experiments were originally modeled using the generalized matching law (Baum, 1974). Davison and McCarthy's (1994) aim was to test the effect of stimulus discriminability on concurrent-schedule performance by varying the wavelengths of the stimuli used for each alternative. In their Part B, the wavelengths used were held constant and were the most discriminable from each other. Thus, to avoid the necessity to account for different discriminability of stimuli, only Part B of their experiment has been analyzed by us. Similarly, we chose Part B of Murrell's (1995) experiment for modeling because that subset had an identical procedure to Davison and McCarthy's (1994) Part B data set and because it was the largest subset of data with homogeneous conditions. Data obtained from these sets were converted into residence time and response rates. Davison and McCarthy's data can be found in the Appendix 
of their article. Murrell's data and description of the experimental conditions, Part B are given in Tables E1 and E2, Appendix E. In both experiments, generalized-matching sensitivity between two alternatives decreased as the reinforcer rate on the third alternative increased. This violates the assumption of generalized matching.

\section{Davison and McCarthy's (1994) Data}

In Part B of Davison and McCarthy's (1994) experiment, three concurrent interdependent schedules were arranged in the random-switching procedures. A response to the switching key would switch all key lights off for a 3-s blackout. After the blackout, one of the three alternatives would be randomly selected with equal probability $(1 / 3)$ and presented to the subject. Overall probability of reinforcement was kept almost constant: 0.022-0.028 per second. Arranged reinforcer ratios of 1:1:9, 9:9:1, 1:1:4, 4:4:1 were implemented for 3 conditions each, so that each reinforcer rate was arranged on each of the 3 keys in different conditions at the same ratio. One condition had reinforcer ratios of 1:1:1. The data from 6 pigeons were averaged over the last five experimental sessions.

Davison and McCarthy analyzed the data on the first and second alternatives in the way that revealed the breach of the generalized matching principle. The data were sorted according to the ratio of $R_{3} /\left(R_{1}+R_{2}\right)$ arranged reinforcer rates. All conditions were divided into two groups: the low-other-reinforcer-rate group if $R_{3} /\left(R_{1}+R_{2}\right)<$ 0.5 and the high-other-reinforcer-rate group if $R_{3} /\left(R_{1}+R_{2}\right)>0.5$. The generalized-matching sensitivities for response rates on the first and second alternatives were consistently higher across subjects in the low-other-reinforcer-rate conditions, in apparent violation of the generalized matching principle.

In our modeling we used both the residence times and response rates, and then assessed how well the models predict the sensitivity values reported in the original article.

\section{Avenues of Analysis Omitted from Presentation}

Because in three-alternative experiments the residence time is not an exact mirror of time allocation, as measured in a 2-key procedure, and is dependent on changeover rate to alternatives, we performed the analysis on both the residence time and the time allocated to alternatives. The latter were calculated from the predicted residence time multiplying by the reported changeover rate from an alternative. The modeling results were practically the same as for the residence time and are not presented here.

It also should be noted that the residence time calculated from Davison and McCarthy (1994) is slightly different from that which is normally used. This is because the appearance of an alternative and time spent responding were triggered probabilistically both by changing over and by the delivery of a reinforcer. However, the delivery of reinforcer was not registered as a changeover; thus the real number of changes between alternatives was 
underestimated proportionally to the reinforcer rate on those alternatives. This caused some increase in the residence time on the more reinforced alternative. We attempted to correct the number of changeovers from an alternative by adding the number of reinforcers multiplied by $2 / 3$, which is the probability of random change to two alternatives other than the current one. The correction did not affect the main result of Navakatikyan's models being better than competing models is not presented here to save space. Similar results were obtained from modeling Murrell's (1995) data.

\section{Davison and McCarthy (1994): Models and Results}

The results of modeling are summarized in Table 1. Two best models to describe response rate were Navakatikyan's powerxhyperbolic-power model with baseline constant (Equation 13) and power×hyperbolic model with baseline constant (Equation 11); the two best models to describe the residence time were also Navakatikyan's powerxhyperbolic-power with baseline constant and hyperbolic xhyperbolic-power with baseline constant functions (Equations $13 \& 12$ ). The models were statistically indistinguishable, i.e. below the chosen cut-off value of 10 . The models' VACs were within $88 \%$ to $93 \%$.

The performance of the competing models was worse, especially for residence time. However, the largest difference between the models was in their prediction of generalized-matching sensitivities. The best of Navakatikyan's models predicted the sensitivities with VAC within $85 \%$ to $98 \%$, while the value for the models based on generalized matching were negative; i.e., the models predicted the obtained sensitivities worse than would a horizontal line drawn through the sensitivity values at their mean.

One of Navakatikyan's models, the hyperbolicxhyperbolic function without a baseline constant, exhibited performance worse than that of Davison and Hunter's model in describing response rate, while the same model with baseline constant outperformed the Davison and Hunter model. Over the full set of 20 Navakatikyan's models

(Table A3, Appendix A), the exponential×hyperbolic function without a baseline constant also performed worse than Davison and Hunter's model; and its performance was greatly enhanced by an addition of the baseline constant. This indicates the importance of the baseline constant, at least for some experiments.

\section{PLEASE INSERT TABLE 1 ABOUT HERE}

Fig. 2 gives an example of response rate and residence time prediction by the best (Navakatikyan, 2007) models alongside Davison and Hunter's and Herrnstein's models for Bird 104, which has values of VACs closest to 
the mean values. The inaccuracy of the generalized matching models' prediction is noticeable, especially for the "second" and "third" alternative.

\section{PLEASE INSERT FIGURE 2 ABOUT HERE}

Fig. 3 shows the predictions for generalized matching sensitivities. Herrnstein's model predicts sensitivity to equal 1 and is obviously inadequate. Davison and Hunter's model predicts sensitivities approximately halfway between the observed values, while Navakatikyan's (2007) predictions are very close to the observed values, especially when those predictions are based on residence times.

\section{PLEASE INSERT FIGURE 3 ABOUT HERE}

\section{Murrell's (1995) Data}

Murrell (1995) aimed to replicate and extend the effects reported by Davison and McCarthy (1994) in a very similar procedure. He kept the reinforcer-rate ratio constant at 1:6 or 6:1 in different subsets of conditions, and gradually increased relative reinforcer rate on the third alternative. Overall reinforcer rate was always at 1.333 reinforcers per minute arranged. In Part B, a blackout of $3 \mathrm{~s}$ following a switch response was arranged, as in Davison and McCarthy. There were 10 conditions in total, in 5 pairs of similar conditions with the same relative reinforcer rate on the third alternative (i.e., 0, 0.13, 0.30, 0.47 and 0.65), see Table E1, Appendix E.

Five values of generalized-matching sensitivity between the first and the second alternatives were calculated for each pair of similar conditions. These values progressively decreased, for both response and time allocation, with increases in the relative reinforcer rate on the third alternative, very closely resembling the prediction from Fig.1C.

\section{Murrell (1995): Models and Results}

The results of the model analyses were very similar to those for Davison and McCarthy's (1994). The best model for response rate was Navakatikyan's power×hyperbolic with baseline constant function (Equation 11), similar to the best for Davison and McCarthy's data. Residence time was described best by the same pair of Navakatikyan's models: powerxhyperbolic-power with baseline constant and hyperbolic xhyperbolic-power with baseline constant (Equations $13 \& 12$ ) as for Davison and McCarthy's data, but in reverse order. These two models 
again were statistically indistinguishable with $\triangle \mathrm{AICc}=1$ only. The models predicted behavior with VACs within $94 \%$ to $95 \%$. The performance of the competing models was substantially worse, especially for residence time. Again, the largest difference between the models was in their prediction of generalized matching sensitivities. The best Navakatikyan's models predicted the sensitivities with VAC within $75 \%$ to $94 \%$, while the values for the models based on generalized matching were negative.

Fig. 4 shows the obtained response rates and residence times, and the predictions by the best of Navakatikyan's models alongside with the competing models'. As reinforcer rate on the third alternative increased, the response rates and residence times on that alternative increased accordingly, while decreasing on the first (richer than the second) alternative, and changing little on the second (poor) alternative. Navakatikyan's model captures the change quite well. Accordingly, it captures the change in the generalized matching sensitivities (Fig. $5)$.

PLEASE INSERT FIGURE 4 ABOUT HERE

\section{PLEASE INSERT FIGURE 5 ABOUT HERE}

\section{Modeling 2: Independent Concurrent VI VI VI Schedules, Steady State Data}

The next two data sets (Davison \& Hunter, 1976; Pliskoff \& Brown, 1976) were from three-alternative experiments with independent concurrent schedules, and produced results consistent with generalized matching.

\section{Davison and Hunter's (1976) Data}

In Davison and Hunter's (1976) experiment, unlike in the other data sets used here, the authors did not use a switching-key procedure; each response key had an associated VI schedule. Extensive response data were obtained from pigeons responding on VI schedules arranged on three, two, and one response keys. Reported data were averaged over pigeons, and used for modeling. There were 5 sets of conditions, that can be described by the association of a schedule with the first, the second and the third key as follows: (1) X, 120 and 60; (2) 60, 120, X; (3) EXT, 120, X; (4) X, 120, EXT; (5) X, EXT, EXT. X denotes a varied VI schedule ranging from extinction to $480 \mathrm{~s} ; 120$ and 60 are VI $120 \mathrm{~s}$ and VI $60 \mathrm{~s}$ (constant within a set) of conditions; and EXT is extinction. 
The following analysis was conducted by Davison and Hunter (1976). Generalized matching sensitivity was calculated for 6 subsets of data: (1) between the VI X s and VI $120 \mathrm{~s}$ alternatives in Set 1; (2) between the VI X s and VI $60 \mathrm{~s}$ alternatives in Set 1; (3) between the VI X s and VI $120 \mathrm{~s}$ alternatives in Set 2; (4) between the VI X s and VI $60 \mathrm{~s}$ alternatives in Set 2; (5) between the VI X s and VI $120 \mathrm{~s}$ alternatives in Set 3; and (6) between the VI $\mathrm{X} \mathrm{s}$ and VI $120 \mathrm{~s}$ alternatives in Set 4. Alternatives with extinction within series were not included in the analysis. The resulting values were similar for all 6 generalized matching sensitivities obtained from group data, ranging from 0.63 to 0.73 for response rates, and from 0.73 to 0.85 for time spent on alternatives.

Additional analysis of log response ratios between constant alternatives (VI $120 \mathrm{~s}$ and VI $60 \mathrm{~s}$ ) in Sets 1 and 2, plotted against arranged reinforcer rate on the alternative with varying VI schedule, did not reveal any trends (Fig. 3, Davison \& Hunter, 1976).

Analysis of the absolute response rate for all 5 sets was also performed, using Herrnstein's model and Davison and Hunter's model $B_{1}=B_{\max }\left[R_{1} /\left(R_{1}+R_{\text {oth }}+k\right)\right]^{s}$ (Model 3, Table A1, Appendix A). The major result was that, while values of $B_{\max }$ from Herrnstein's model varied substantially between the keys, ranging from 70 to 325 , while $B_{\max }$ from Davison and Hunter's Equation 14 ranged only from 70 to 125 and all, except one, values of sensitivities $a$ were between 0.62 and 0.82 .

Thus Davison and Hunter's models confirmed the presence of undermatching, independence of response-rate ratios from other alternatives and the superiority over strict matching, i.e. the principle of generalized matching.

The present analysis took data from Davison and Hunter's Table 1, which shows the data averaged across subjects. Since they did not present any switching data, only response rates could be extracted for these analyses.

\section{Davison and Hunter (1976): Models and Results}

Table 1 contains our reanalysis in terms of the Law of Effect models under consideration. There are three models within the cutoff value of $\triangle \mathrm{AICc}=10$, two of Navakatikyan's type, and Davison and Hunter's model. These three models showed quite high VACs for predicted response rates, between 96 and 97\%. We could have considered a more liberal cutoff value of $\triangle \mathrm{AICc}=6$ (Burnham \& Anderson, 1998) to narrow the list of the models, and in this case two of Navakatikyan's models would be the best. However, three of the other Navakatikyan models are worse than both Davison and Hunter's model and Herrnstein's model. Thus we cannot conclude that Navakatikyan's models are unambiguously better, but neither is Davison and Hunter's.

Because there was little variation in sensitivity values, we could not meaningfully evaluate the VAC of the models' predictions of sensitivity as we did for the other experiments reanalyzed here. (Recall that VAC effectively 
compares the error of prediction with that of the error of assuming that all values are equal to their mean.) We therefore emphasize the prediction of log response ratios for all pairs of alternatives, except extinction schedules. These VACs were high, ranging from $92 \%$ to $94 \%$ for the best three models (Table 1).

The fits and data are plotted in Fig. 6. Sets of similar conditions (i.e., Set 1 and 2, and Set 3 and 4) are joined in the figure, because the models without bias were the better models and we can therefore ignore the location of an alternative and concentrate on the arranged schedule. The response rate for each schedule was plotted against varying-schedule reinforcer rate, and the sets of conditions were positioned vertically, so the values of similar schedules can be compared horizontally. The best Navakatikyan's model performs slightly better than the other models, especially at higher arranged reinforcer rates (upper row of panels, Fig. 6). Oddly, no model described response rates well when there were extinction schedules arranged on both Keys 2 and 3. It is difficult to explain why the maximum response rate of a single reinforced alternative seems lower than in conditions with only one or no extinction schedule. However, since the conditions in question were conducted after those with one or no extinction alternatives, it may be that some overall time-dependent trend in behavior is responsible. Thus, we are not sure how much weight to give to this finding.

\section{PLEASE INSERT FIGURE 6 ABOUT HERE}

The predictions for the generalized matching sensitivity are given in Fig. 7. As mentioned above, there was little variation in the sensitivity data. It is clear that both Davison and Hunter's model and Navakatikyan's model were predicting values consistent with the generalized matching, i.e., close to equal sensitivity.

\section{PLEASE INSERT FIGURE 7 ABOUT HERE}

\section{Pliskoff and Brown's (1976) Data}

The next data set was provided by Pliskoff and Brown's (1976) Table 2. They used a three-alternative switching-key procedure similar to Davison and McCarthy's (1994) and Murrell's (1995). However, in Pliskoff and Brown's experiment, there was no blackout between switches, but there was a changeover delay of $1.5 \mathrm{~s}$. A peck on the switching key would randomly select any alternative with equal probability of $1 / 3$. Three VI tapes apparently ran independently (although this is not explicitly stated in their paper). Overall arranged reinforcer rate was kept 
constant at 0.75 reinforcers per minute. Nine conditions were conducted, including one with extinction on one alternative, and two with extinction on two alternatives. Pliskoff and Brown concluded that "matching occurs in much the same fashion as with two schedules" (p. 73), but 3 of their 6 data sets ( 3 pigeons, and both relative time and relative response rates) showed undermatching rather than strict matching.

Davison and McCarthy (1988) reanalyzed Pliskoff and Brown's (1976) data, and calculated generalizedmatching sensitivities for each pair of alternatives, excluding those that arranged extinction (Davison \& McCarthy's Table 7.6, p. 111). Davison and McCarthy concluded that the data were consistent with generalized, rather than strict, matching.

In order to model the data, we took Pliskoff and Brown's (1976) response rate data and derived the residence time values. In two conditions, Bird 11 did not respond on the extinction alternatives, and to enable modeling the residence time on these alternatives data were Winsorized (i.e., replaced by the next most extreme value. In this case, a value of $6000 \mathrm{~s}$ was replaced by $86.47 \mathrm{~s}$, and a value of $0 \mathrm{~s}$ by $0.39 \mathrm{~s}$ ).

\section{Pliskoff and Brown (1976): Model and Results}

The results of modeling are shown in Table 1, Fig. 8 and Fig 9. There are three models for response rate in the confidence set of the best models, two of Navakatikyan's models (hyperbolicxhyperbolic-power function with baseline constant and hyperbolicxhyperbolic model) and Stevens' model. These three models' VACs were close, between $95 \%$ and $98 \%$. Fits for other models were similar, with VAC ranging from $93 \%$ to $97 \%$. The model ranked lowest by $\triangle \mathrm{AICc}$ was Herrnstein's model but its VAC value was high at $93 \%$. Note that a good performance of Stevens' model shows that reinforcer rates were arranged in insufficiently independent fashion - as the reinforcer rate on other than the current alternative is not required for this model. Predictions for response rate, as well as for residence time, for Herrnstein's, Davison and Hunter's and the best of Navakatikyan's models (hyperbolicxhyperbolic-power function with baseline constant) are shown in Fig. 8.

Predictions for generalized-matching sensitivities for the best of Navakatikyan's and Davison and Hunter's models were reasonable, with VACs of 55\% and 45\% respectively, and similar values for all three pairs of alternatives (Fig. 9). Herrnstein's model was not successful, giving a negative value of VAC. As there was little variation in the data, high VACs could not be expected, as discussed earlier. A more dependable conclusion about the accuracy of generalized matching can be obtained from the VACs for log ratios of response rate. Those were all high, between 95\% and 97\%, except for $83 \%$ for Herrnstein's model (the values are omitted from Table 1). 
Thus, the results for the modeling confirmed the consistency of the response-rate data with the generalizedmatching principle.

\section{PLEASE INSERT FIGURE 8 ABOUT HERE}

The models for residence time led to completely different conclusions, however, demonstrating the superiority of the Navakatikyan models (see Fig. 8). All six of them ranked higher than any other model. The best model's VAC was 95\%, while VACs of the Davison and Hunter model and the Herrnstein model were $75 \%$ and 81\%. Generalized-matching sensitivities were also predicted differently; all of Navakatikyan's models had VACs positive, between 18 and 59\%, except for one (hypxhyp, VAC $=-248 \%$ ), while Davison and Hunter's model's VAC was $-594 \%$ and Herrnstein's was $-348 \%$. VACs for the log residence time ratio for Navakatikyan's models were between 92 and 97\%, while the competing models' VACs were between 82 and $88 \%$ (the values are omitted from Table 1).

Plots of the generalized matching sensitivities for the residence time data show orderly increasing trends across the different pairs of alternatives (Fig. 9). Navakatikyan's models, predict these increases, but Davison and Hunter's and Herrnstein's models predict constant sensitivities.

We decided to check whether the values of sensitivity depended on two factors: birds and pair of alternatives for which the sensitivity was calculated. We applied two-factor ANOVA to the response rate, time spent, and residence time sensitivities. The results are as follows. (A) Response rate; the $F$-test for regression does not provide evidence against the hypothesis that none of the factors, i.e., birds or pairs of alternatives, are related to generalized-matching sensitivity $(p=.125)$. This is consistent with the generalized-matching principle. (B)

Residence time and time spent; the $F$-test provides weak evidence against the hypothesis that none of the factors are related to sensitivity ( $p=.052$, for both residence time and time spent), and there is weak evidence that for residence time, as well as for time spent, both birds and pairs of alternatives affect the value of sensitivity $(p=.059$, for each of the variables). The latter result is not consistent with the generalized-matching principle, and that might be the reason for the superiority of Navakatikyan's models in describing residence times.

\section{PLEASE INSERT FIGURE 9 ABOUT HERE}




\section{Modeling 3: Interdependent Concurrent VI VI VI VI Schedules, Dynamical Data}

\section{Elliffe and Davison's (2010) Data}

The final data set came from Elliffe and Davison (2010). They used a four-alternative rapidly changing procedure that is similar to the two-alternative procedure used by Davison and Baum (2000). As in Davison and Hunter's (1976) experiment, each response key had an associated VI schedule, but the association changed each 10 reinforcers.

Elliffe and Davison's (2010) rapidly-changing procedure contained 4 reinforcer rates that were randomly allocated to each of the alternatives for a component of 10 reinforcers on a 4-key concurrent VI schedule. The reinforcers were scheduled dependently and formed a 27:9:3:1 reinforcement ratio with an overall arranged reinforcer rate of 3 reinforcers per min. After the subjects received 10 reinforcers, there was a blackout for $10 \mathrm{~s}$ and then the reinforcer rates were pseudorandomly reallocated between keys for the next component. Each experimental session contained 8 components; therefore the reinforcer rates were allocated 8 times per session. There were 6 pigeons in the experiment. Elliffe and Davison modeled their data using a many-alternative version of the generalized matching law (see Schneider \& Davison, 2005) and showed that overall sensitivity increased with each successive reinforcer in a component, thus their data violated the generalized-matching principle, suggesting that model was inappropriate.

For the present analysis, the response data for each reinforcer rate were subdivided into four groups. Data were grouped in 16 (4-by-4) possible combinations of previous and current reinforcer rates on an alternative, and models for response rate and changeover rate were built. In this analysis, we modeled all three major behavioral indices: the response rate, the residence time, and the changeover rate; and calculated the fourth index - time spent per min on each alternative - from the predicted residence time and changeover rate. We substituted the commonly used index, time spent, by time spent per minute in order to adjust for different length of sessions and to present data graphically. Such a substitution does not influence the calculation of time-allocation sensitivity. We also calculated the time spent per min on each alternative as a product of the predicted residence time and observed changeover rate.

The generalized matching point-sensitivity was calculated at each reinforcer in the component, and the accuracy of prediction assessed for each of 4 indices. Unlike in the original article, we calculated the sensitivities for the data averaged over subjects. We present the results of the sensitivity assessment only for 3 of the possible 
reinforcer ratios: 27:9, 9:3 and 3:1 to save space. The results for all 6 pairs, i.e., with 27:3, 27:1 and 9:1, ratios were similar.

For the purpose of presentation and to assess the overall prediction means, all the results were averaged also over the previous four reinforcer rates. This averaging negated the large random variation in residence time compared with the overall trend. The predictions for the time spent were not averaged but calculated from the averages of predicted residence time and changeover rate, and generalized matching sensitivities were also calculated after averaging the reinforcer rate and behavior over birds and over previous reinforcer rates.

\section{Elliffe and Davison (2010): Models and Results}

The results are presented in Table 1. The modeling confirmed the conclusion of Elliffe and Davison (2010) about the inadequacy of generalized matching as a description of these data. All Navakatikyan's models proved to be superior by $\triangle \mathrm{AICc}$ and ranked from $1^{\text {st }}$ to $6^{\text {th }}$, although the variances explained by different models were similar. The models without the baseline constant performed worse, ranking $5^{\text {th }}$ and $6^{\text {th }}$. The Navakatikyan models were also much better in predicting the generalized matching point-sensitivities. The performance of the models for changeover rate and time allocated per minute was very close to that of the response-rate models; and the ranking of the models was the same, thus these results were not presented in Table 1. Accuracy of the residence-time predictions were seemingly low, but the reason for that was the higher random variance in the residence times compared to the general trend, which will be shown on the averaged data. The medians of the time constant for all three behavioral indices were remarkably close, equaling 0.22-0.23 (Table D1, Appendix D).

An example of the response rate fits for Bird 85 is presented in Fig. 10.

\section{PLEASE INSERT FIGURE 10 ABOUT HERE}

Fig. 11 presents the fits averaged over birds and the previous conditions for the best of Navakatikyan's, Davison and Hunter's and Herrnstein's models. Except for the residence time, the VAC is high for all models, ranging between $94 \%$ and $99 \%$, slightly in favor of Navakatikyan's model. However, the plots show the imperfection of fits of the Davison and Hunter and Herrnstein models, despite the small difference in VAC (3\% to $4 \%$ ). The residence-time data produced worse fits, with 83\%, $29 \%$ and 43\% VAC for Navakatikyan's, Davison and Hunter's and Herrnstein's models, respectively. It is worth noting that all three major indices, the response rate, the 
changeover rate and the residence time were increasing functions of the reinforcer rate. (Note that due to the higher level of averaging, the VAC values given in Fig. 11 and Fig. 12 are higher than those in Table 1).

\section{PLEASE INSERT FIGURE 11 ABOUT HERE}

Sensitivities for each behavioral index calculated between alternatives with 27 and 9,9 and 3, 3 and 1 reinforcers per 40 overall reinforcers delivered are given in Fig. 12. If the data were consistent with the generalized matching law we would expect that the curves would plateau and converge to a single value of sensitivity. That was not the case, the curves asymptoted at distinct and different values. The data and model fits for 9:3 and 3:1 ratios have a peculiar shape, first converging to each other, then diverging and stabilizing. That was observed in all modeled variables, and thus has to be attributed to the variations in the reinforcer rate. The accuracy of Navakatikyan's models was high and noticeably higher than that of the other models. Overall, the predictions for the residence time were less accurate, but that is related to the higher level of random variation in the parameter compared to the modeled trend.

\section{PLEASE INSERT FIGURE 12 ABOUT HERE}

\section{Discussion}

The primary goal of this article was to compare the performance of a component-functions model of choice behavior (Navakatikyan, 2007) with the LOE models based on the matching principle - Herrnstein's (1970) and Davison and Hunter's (1976) models - in situations involving choice between more than 2 alternatives.

Navakatikyan's models were represented by 6 equations (Equations 8 to 13) with 3, 4 and 5 parameters, excluding bias. Stevens' (1957) model was added to the analysis in order to test the data for the lack of independence between reinforcer rates on different alternatives. Five data sets from concurrent VI schedules experiments were chosen for modeling: Davison and McCarthy, 1994; Murrell, 1995; Davison and Hunter, 1976; Pliskoff and Brown, 1976; and Elliffe and Davison, 2010.

\section{Overall Performance of the Models}

Overall the performance of Navakatikyan's models according to the Akaike information criterion was superior. There were 10 subsets of data available for the modeling: 5 subsets of response rate, 4 subsets of 
residence time, and 1 subset of changeover rates. In two subsets of response rates, from Davison and Hunter (1976) and Pliskoff and Brown (1976), the Navakatikyan models performed on par with Davison and Hunter's and Herrnstein's models. In all other subsets, all 20 of Navakatikyan's models described in Table A3 (Appendix A) performed better. There was only one exception. In the response-rate subset of Davison and McCarthy's (1994) experiment, two of the simplest models without baseline constant (hyperbolicxhyperbolic and exponentialxhyperbolic) were worse than some competing models. However, if we allow the baseline constant to be non-zero, the models outperformed the competitors (see Table 2).

\section{PLEASE INSERT TABLE 2 ABOUT HERE}

With the exception of Stevens' response-rate model for Pliskoff and Brown's data, none of the competing models passed the test for the absence of trend in the residuals and, with the exception of Davison and Hunter's data; none described GML sensitivity values well.

It might be argued that due to the substantial number of Navakatikyan's models that were tested, a model that is better than competitors will always be found. That is a legitimate concern. To resolve it we performed a two-way ANOVA on models' $\triangle \mathrm{AICc}$ ranks within each subset of data. One factor was Subset, the other was Model group: Navakatikyan's or Competitors. We performed two analyses: with all four competing models and with only the better ones, namely, Davison and Hunter's models (Models $3 \& 4$, Table A1, Appendix A). In both cases the differences between groups of the models were overwhelmingly in favor of Navakatikyan's models $(F(1,229)=95.6$, $p<.0001 ; F(1,209)=38.9, p<.0001)$. Similar results were obtained in previous modeling. In Navakatikyan's (2007) article, all 35 models based on a multiplicative relationship between component functions were better than any of the competing models. In Navakatikyan and Davison's (2010) modeling, the Navakatikyan models were also better or on par with competitors. Thus, it can be stated that accounting for a multiplicative relationship between reinforcers on present and other alternatives and for presence of baseline behavior (i.e., for the structure of Equation 7) gives a model an advantage.

Such a structure is not the only possibility where a variety of component-functions could be encompassed by a main idea. In this study we conducted a limited assessment of a structure of the type $B=F_{\text {enh }}\left(R_{1}\right) \times F_{\text {red1 }}\left(R_{\text {oth1 } 1}\right) \times$ $F_{\text {red2 }}\left(R_{\text {oth2 }}\right)+B_{\mathrm{a}}$ (with a separate reducing-component function for reinforcers on each of other alternatives, unlike in Equation 7). For the subsets of response rates the results were ambiguous, but for the residence time Equation 7 
was always the better choice. An additive structure, $B=F_{\text {enh }}+F_{\text {red }}+B_{\mathrm{a}}$, or a structure similar to Davison and Hunter's model, $B=F\left(R_{1}\right) /\left[\sum\left(R_{\mathrm{i}}\right)+k\right]$ with arbitrary function $F(R)$ can be explored in the future.

Though there were many of Navakatikyan's models tested alongside of the few competing models, it is important to recognize that were are at least 8 of Navakatikyan's response rate models (placed in Appendix A, Table A4, $1^{\text {st }}$ to $6^{\text {th }}$ and $8^{\text {th }}$ to $9^{\text {th }}$ from above, e.g., Equation 13) that were always better or in two cases on par with any competing models; while all of Navakatikyan's models for resident time and changeover rate were better than any competing model (see Table A4).

\section{Which Component-Functions Model is Better?}

For response rate, performance of the models with 5 and 4 free parameters were on average similar: models based on hyperbolic enhancing-component function (hyp $\times$ hypp $+b$, hyp $\times h y p+b$ ) performed better for Davison and Hunter's (1976), Pliskoff and Brown's (1976), and Elliffe and Davison's (2010) data, while models based on power enhancing-component function (pow $x h y p+b$, pow $x h y p p+b$ ) performed better for Davison and McCarthy's (1994), and Murrell's (1995) data. For residence time or time spent per minute on each alternative, two models with 5 free parameters were the best by all performance indicators: they have the lowest $\triangle \mathrm{AICc}$, they pass the test for trend in residuals and they describe changes in GML sensitivities very well. Although we investigated all 20 Navakatikyan's models shown in Table A3, we have reported detailed results (Table 1) of those analyses for only those 6 models that were the best for at least some experiments (or were simplifications of those best models). There was no consistently best model across all experiments, either for residence time or for response rate (Table A4). For residence time, the pow $\times$ hypp $+\mathrm{b}$ model was also the best model as defined by total sum of $\Delta$ AICc. For response rate, the best model by total sum of $\triangle \mathrm{AICc}$ was Model 20 from Table A3 (expxdlogt $+\mathrm{b}$ ), but we have not included this model in the detailed analyses because it was not the best model for any experiment. Our purpose is not to identify the best pair of enhancing- and reducing-component functions - the data don't allow us to do this

- but to demonstrate the superiority of the structure of Navakatikyan's approach to the competing LOE models, and to show that there are several pairs of component functions that perform particularly well.

For all data sets, none of the models we analyzed proved to be a better fit with added bias parameters than without. This is not to say that there was no bias in any data set. Rather, it implies that any bias was too small to justify, according to AICs, adding 2 bias parameters to a model for 3-alternative choice, or 3 parameters to a 4choice model. It seems certain that experiments investigating choice between multiple alternatives that arranged 
different operanda, or force requirements, for example, would require bias parameters and our analysis should not be taken to imply that bias can be ignored.

Initially, when the main concept of Navakatikyan's model was presented at the New Zealand Association for Behaviour Analysis Symposium (Auckland, New Zealand, 1998) and later at the Association for Behavior Analysis annual convention (Chicago, 1999), it was thought that both enhancing- and reducing-component functions could be of the same type, namely, a logistic function. However, in his reanalysis of 2-alternative choice data, Navakatikyan (2007) found that the best models for residence time were functions with 6 free parameters, including bias, and that the single best model out of 35 was a power $x$ hyperbolic model with baseline constant and bias. There were some minor differences between Navakatikyan's modeling and that reported here. In the previous analysis, the enhancing-component power function was of the form $a+b R^{c}$ (i.e., with an extra additive constant $a$ ). Navakatikyan did not assess the reducing-component hyperbolic-power function that we used here, and used a simple hyperbolic decreasing function instead. Navakatikyan and Davison (2010) used a simple 4parameter hyperbolic xhyperbolic model, without a baseline constant but with bias, because the main goal was to demonstrate the superiority of the principle and because the scarcity of data favored a simple model.

In the analysis reported here, different component functions perform better for different experiments. Our initial conjecture that for an enhancing-component function an unbounded power function would be more suitable for residence time description, while a hyperbolic function would be better for the response rate, proved to be wrong. Thus, we are not able to formulate the results in terms of a universally best model. In general, we are now not convinced that a single pair of enhancing and reducing functions will exist that will describe the data from all experiments with sufficient accuracy. To the contrary, as the procedures and contingencies differ between experiments, we might expect the models that best describe them also to differ. It is noteworthy that the residencetime models for Davison and McCarthy's (1994) and Murrell's (1995) data are very similar and predict GML sensitivities with high accuracy, given that these two experiments are very similar in procedure and design.

Could we glimpse some idea on what component-functions ought to be for different schedules? Our best hope would be to follow along the lines of Killeen's mathematical principles of reinforcement (Killeen, 1994). However, there are two obstacles. First, we cannot use as enhancing-component functions the equations derived by Killeen for single-response schedules, as one of his main assumptions - the assumption of randomly emitted responses - does not hold for concurrent schedules. In concurrent schedules responses are emitted in sequences allocated to alternatives. If they were emitted randomly, say, in a two-alternative concurrent VIVI schedule, then 
the inter-response times (IRT) and residence times (RT) on opposite alternatives should come from the same exponential distribution. We tested this on data from Alsop and Elliffe (1988) and from Elliffe and Alsop (1996) using $F$-statistics (e.g., Weerahandi, 1995, p.38-39). The results are overwhelmingly against the null-hypothesis, that those time measures came from the same distribution. Out of 750 RT/IRT ratios obtained in these two studies ( 2 studies $\times 6$ birds $\times$ about 30 conditions $\times 2$ alternatives for each condition), in 749 ratios the residence time was significantly larger than inter-response time ( $p$-values $<0.01$ ). The minimum value of IRT/RT-ratio was 1.9 , with median value equal to 12.7. Second, similarly to Killeen's approach we will need a feedback function for our derivations. To the best of our knowledge, only feedback functions for two-alternative concurrent schedules without a changeover delay are derived (Heyman \& Luce, 1979). These are required to solve 4 simultaneous equations for probabilities and rates in equilibrium state of Markov chain. Similar functions for three-alternative concurrent interdependent concurrent schedule involve solutions to 9 equations (Wiremu Solomon, personal communication) and for the four-alternative schedule, solutions to 16 equations. This also disregards the complicating effects of changeover delays on feedback functions. Such an analysis goes beyond the scope of the present paper, and probably beyond our mathematical ability.

\section{The Matching Principle}

Navakatikyan's (2007) models were most successful in describing the experiments where it was already independently established that data are not consistent with generalized matching. From the beginning of the analysis we knew that Davison and McCarthy's (1994), Murrell's (1995) and Elliffe and Davison's data (2010) contradict generalized matching, and that Navakatikyan's models predict a decrease in GML sensitivity between two alternatives when the reinforcer rate is increased on the third alternative. The modeling confirmed that Navakatikyan's models describe sensitivity in these experiments very well.

All the models we assessed, including Navakatikyan's (2007) models, performed well with Davison and Hunter's (1976) data, even though those data are consistent with generalized matching. This is important, because it shows that Navakatikyan's models are capable of describing data that show varying degrees of consistency with generalized matching. Also, while no model described Pliskoff and Brown's (1976) residence-time sensitivity data very well, Navakatikyan's models all did so much better than any of the competing models, probably due to their substantially more accurate prediction of residence time (VAC for the residence time predictions was 94 to $95 \%$ for all 6 Navakatikyan's models vs. 75\% for Davison and Hunter's model and $-81 \%$ for Herrnstein's, see Table 1).

Pliskoff and Brown's data are also not obviously inconsistent with generalized matching, perhaps because they are 
quite variable. The implication is that models that assume generalized matching, or that are built on generalizedmatching principles, do not necessarily describe better even those data that are consistent with generalized matching. Overall, Navakatikyan's models describe multiple-alternative choice at least as well as their competitors, whether or not those data are consistent with generalized matching.

\section{Independent vs. Interdependent Schedules: VI Feedback Functions}

There are two peculiarities in our results that we want to mention although we are not sure of their origin. The first peculiarity is that the advantages of Navakatikyan's (2007) models were comparatively smaller for Davison and Hunter's (1976) and Pliskoff and Brown's (1976) data sets (though overall the models' performance was high). It is possible, that the design of other experiments was built around keeping constant a reinforcer rate ratio between two alternatives, while varying reinforcer rate on the other alternatives, which is proved to violate substantially the generalized matching principle and provide an advantage to Navakatikyan's models. But could the reason lie with the difference between independent and interdependent VI schedules? Could the independent schedule be conducive to generate data with smaller interaction between behavioral alternatives? We think that there are some indications of this. For example, Stevens' (1957) model, which assumes no interaction between reinforcer rates on different alternatives, was part of the confidence set of models in Pliskoff and Brown's response rate data set.

The second peculiarity is that Navakatikyan's models were uniformly successful in describing residence time or time spent per minute on each alternative, while response-rate data presented a more ambiguous picture. We are tempted to speculate that the residence-time data are better material for Navakatikyan's models because the residence times on alternatives affect reinforcer rate through VI feedback functions (e.g., Heyman \& Luce, 1979). At the same time response rate affects reinforcer rate indirectly, through residence time, and thus brings some statistical randomness into the data, assuming that there is some variability in response rate. We think that this feature of the VI schedules might explain the well-known but not understood fact that GML sensitivities for time allocation are generally higher than response allocation (e.g., Alsop \& Elliffe, 1988; Baum, 1979; Elliffe \& Alsop, 1996; Taylor \& Davison, 1983). If this is so, the sensitivity of the time ratio should be higher because it drives reinforcer rate on each alternative more directly.

In summary, Navakatikyan's (2007) component-functions models for the Law of Effect proved to be usually better and at least on par with the models consistent with the generalized-matching principle in describing choice between more than two concurrently arranged VI schedules. The superiority of Navakatikyan's models is most 
marked when modeling residence time, but is also clearly visible to a lesser degree when modeling response rate. Navakatikyan's models can describe a decrease in GML sensitivity between two alternatives when the reinforcer rate is increased on the third alternative, thus explaining the systematic inconsistencies with the generalized matching principle in the analyzed data sets.

More generally, the modeling reported here add to the growing evidence that, although the generalizedmatching relation usually describes choice reasonably well, the principle or mechanism that underlies choice cannot be generalized matching (e.g., Elliffe, Davison \& Landon, 2008). Whether or not each data set was adequately described by the generalized matching law, Navakatikyan's (2007) models, which explicitly do not assume generalized matching, described GML sensitivity and how it may change better than did models built on the generalized-matching principle. 


\section{REFERENCES}

Ainsworth, S. (1977). Steady-state enzyme kinetics. London: Macmillan Press.

Allen, G. M. (1981). On the exponent in the "Generalized" matching equation. Journal of the Experimental Analysis of Behavior, 35, 125-127.

Allen, G. M. (1982). Comment on Houston's argument. Journal of the Experimental Analysis of Behavior, 38, 1131114.

Alsop, B., \& Elliffe, D. (1988). Concurrent-schedule performance: Effects of relative and overall reinforcer rate. Journal of the Experimental Analysis of Behavior, 49, 21-36.

Baum, W. M. (1974). On two types of deviation from the matching law: Bias and undermatching. Journal of the Experimental Analysis of Behavior, 22, 231-242.

Baum, W.M. (1979). Matching, undermatching, and overmatching in studies of choice. Journal of the Experimental Analysis of Behavior, 32, 269-281.

Boelens, H., Kop, P. F. M., Nagel, A. L., \& Slangen, J. L. (1987). Concurrent schedules: Effects of reinforcement rate and changeover delay on time allocation in a three-alternative procedure. The Quarterly Journal of Experimental Psychology, 39B, 229-244.

Burnham, K. P., \& Anderson, D. R. (1998). Model selection and multimodel inference: A practical information theoretic approach. (2nd ed.). New York: Springer-Verlag.

Davison, M. (1982). Preference in concurrent variable-interval fixed-ratio schedules. Journal of the Experimental Analysis of Behavior, 37, 81-96.

Davison, M., \& Baum, W. M. (2000). Choice in a variable environment: every reinforcer counts. Journal of the Experimental Analysis of Behavior, 74, 1-24.

Davison, M., \& McCarthy, D. (1994). Effects of the discriminabilty of alternatives in three-alternative concurrentschedule performance. Journal of the Experimental Analysis of Behavior, 61, 45-63.

Davison, M. C., \& Hunter, I. W. (1976). Performance on variable-interval schedules arranged singly and concurrently. Journal of the Experimental Analysis of Behavior, 25, 335-345.

Elliffe, D., \& Alsop, B. (1996). Concurrent choice: Effects of overall reinforcer rate and the temporal distribution of reinforcers. Journal of the Experimental Analysis of Behavior, 65, 445-463.

Elliffe, D., \& Davison, M. (2010). Four-alternative choice violates the constant-ratio rule. Behavioural Processes, 84, 381-389. 
Elliffe, D., Davison, M., \& Landon, J. (2008). Relative reinforcer rates and magnitudes do not control concurrent choice independently. Journal of the Experimental Analysis of Behavior, 90, 169-185.

Herrnstein, R. J. (1970). On the law of effect. Journal of the Experimental Analysis of Behavior, 13, 243-266.

Herrnstein, R. J. (1974). Formal properties of the matching law. Journal of the Experimental Analysis of Behavior, $21,159-164$.

Herrnstein, R. J., \& Loveland, D. H. (1975). Maximizing and matching on concurrent ratio schedules. Journal of the Experimental Analysis of Behavior, 24, 107-116.

Herrnstein, R. J., \& Vaughan, W., Jr. (1980). Melioration and behavioral allocation. In J. E. R. Staddon (Ed.), Limits to action: The allocation of individual behavior. New York: Academic Press.

Heyman, G. M. \& Luce, R. D. (1979) Operant matching is not a logical consequence of maximizing reinforcement rate. Journal of the Experimental Analysis of Behavior, 79, 133-140.

Horner, J. M., \& Staddon, J. E. R. (1987). Probabilistic choice - a simple invariance. Behavioural Processes, 15, $59-92$.

Jensen, G., \& Neuringer, A. (2009). Barycentric extension of generalized matching. Journal of the Experimental Analysis of Behavior, 92, 139-159.

Killeen, P. R. (1994). Mathematical principles of reinforcement. Behavioral and Brain Sciences. 17, 105-172.

Lobb, B., \& Davison, M. C. (1975). Performance in concurrent interval schedules: A systematic replication. Journal of the Experimental Analysis of Behavior, 24, 191-197.

Luce, R. D. (1959). Individual choice behavior: A theoretical analysis. New-York: Willey.

Luce, R. D. (1977). The choice axiom after twenty years. Journal of Mathematical Psychology. 15, 215-233.

Mazur, J. E. (1992). Choice behaviour in transition: Development of preference with ratio and interval schedules. Journal of Experimental Psychology: Animal Behavior Processes, 18, 364-378.

Mazur, J. E., \& Ratti, T. A. (1991). Choice behavior in transition: Development of preference in a free operant procedure. Animal Learning and Behaviour, 19, 241-248.

McDowell, J. J. (1986). On the falsifiability of matching theory. Journal of the Experimental Analysis of Behavior, $45,63-74$.

Miller, H. L., \& Loveland, D. H. (1974). Matching when the number of response alternatives is large. Animal Learning and Behaviour, 2, 106-110. 
Murrell, P. R. (1995). Choice on three concurrent alternatives. Unpublished MSc thesis, The University of Auckland, New Zealand.

Navakatikyan, M. A. (2007). A model for residence time in concurrent variable interval performance. Journal of the Experimental Analysis of Behavior, 87, 121-141.

Navakatikyan, M. A., \& Davison, M. (2010). The dynamics of the law of effect: A comparison of models. Journal of the Experimental Analysis of Behavior, 93, 91-127.

Pliskoff, S. S., \& Brown, T. G. (1976). Matching with a trio of concurrent variable-interval schedules of reinforcement. Journal of the Experimental Analysis of Behavior, 25, 69-73.

Prelec, D. (1984). The assumptions underlying the generalized matching law. Journal of the Experimental Analysis of Behavior, 41, 101-107.

Schneider, S. M., \& Davison, M. (2005). Demarcated response sequences and generalised matching. Behavioural Processes, 70, 51-61.

Stevens, S. S. (1957). On the psychophysical law. The Psychological Review, 64, 153-181.

Taylor, R., \& Davison, M. (1983). Sensitivity to reinforcement in concurrent arithmetic and exponential schedules. Journal of the Experimental Analysis of Behavior, 39, 191-198

Vaughan, W., Jr. (1981). Melioration, matching, and maximization. Journal of the Experimental Analysis of Behavior, 36, 141-149.

Vaughan, W., Jr, \& Miller, H. L. J. (1985). Choice: A local analysis. Journal of the Experimental Analysis of Behavior, 43, 337-348.

Weerahandi, S. (1995). Exact Statistical Methods for Data Analysis. New York: Springer-Verlag.

White, A. J., \& Davison, M. C. (1973). Performance in concurrent fixed-interval schedules. Journal of the Experimental Analysis of Behavior, 19, 147-153. 


\section{AUTHOR NOTE}

We acknowledge the contributions to this work by Mark Stewart, Wiremu Solomon and Brian McArdle. 
Table 1

Comparison of the models' performance across sets of the data. DM, Mur, DH, PB, ED refer to Davison and McCarthy's (1994), Murrell's (1995), Davison and Hunter's (1976), Pliskoff and Brown's (1976), and Elliffe and Davison's (2010) data. Models' abbreviations: hyp xhyp, hyp xhyp + b, hyp×hypp + b refer to Navakatikyan's (2007) hyperbolic-hyperbolic without baseline constant, with baseline constant, and hyperbolicxhyperbolic-power with baseline constant models, respectively (Equations 8,9 \& 12); pow $\times$ hyp, pow $\times$ hyp $+b$, power $\times$ hypp $+b$ are power-hyperbolic without baseline constant, with baseline constant, and powerxhyperbolic-power with baseline constant models (Equations 10, 11 \& 13); Stevens, Herrnstein and Davison-Hunter are Stevens's (1957), Herrnstein's (1970) and Davison-Hunter's (1976) models (Equations 3 to

5). VAC is percentage of variance accounted for. $\triangle \mathrm{AICc} \leq 10$ indicates models within a confidence set. CSet is confidence set, i.e. number of cases when model was in the confidence set. Asterisk (*) flags data sets with no statistically significant trend in residuals in all or most of the subjects. Sum is sum of $\Delta$ AICc, i.e. the models are ordered by the sums of values of $\triangle \mathrm{AICc}$ across experiments.

\begin{tabular}{|c|c|c|c|c|c|c|c|c|c|c|c|c|c|c|c|c|c|c|c|c|}
\hline \multirow{3}{*}{ Model } & \multirow{3}{*}{$m$} & \multicolumn{7}{|c|}{$\triangle A I C c$} & \multicolumn{6}{|c|}{$\begin{array}{l}\text { VAC for behavioral index (\%) and flag of } \\
\text { passed test for trend in residuals }(*)\end{array}$} & \multicolumn{6}{|c|}{ VAC of GML sensitivity or log ratios } \\
\hline & & \multicolumn{5}{|c|}{ Data } & \multirow{2}{*}{ Sum } & \multirow{2}{*}{ CSet } & \multicolumn{5}{|c|}{ Data } & \multirow{2}{*}{ Mean } & \multicolumn{5}{|c|}{ Data } & \multirow{2}{*}{ Mean } \\
\hline & & $D M$ & Mur & $\mathrm{DH}$ & $P B$ & $E D$ & & & $D M$ & Mur & $\mathrm{DH}$ & $P B$ & $E D$ & & $D M$ & Mur & $\mathrm{DH} \dagger$ & $P B$ & $E D$ & \\
\hline \multicolumn{21}{|c|}{ Modeling of response rate } \\
\hline hyp xhypp + b & 5 & 44 & 29 & 1 & 0 & 0 & 74 & 3 & $* 86$ & $* 94$ & *97 & $* 98$ & $* 94$ & 93 & 60 & 69 & 94 & 55 & 87 & 73 \\
\hline hypxhyp + b & 4 & 66 & 16 & 0 & 11 & 3 & 96 & 2 & 84 & *94 & *97 & 97 & $* 94$ & 93 & 63 & 64 & 93 & 16 & 87 & 64 \\
\hline pow $\times$ hyp + b & 4 & 5 & 0 & 53 & 20 & 25 & 104 & 2 & $* 88$ & $* 94$ & 93 & *95 & $* 93$ & 93 & 90 & 75 & 77 & 38 & 85 & 73 \\
\hline pow $\times$ hypp $+b$ & 5 & 0 & 25 & 56 & 26 & 15 & 122 & 1 & $* 88$ & $* 94$ & 93 & $* 95$ & $* 94$ & 93 & 85 & 75 & 78 & 48 & 86 & 74 \\
\hline pow $\times$ hyp & 3 & 65 & 80 & 52 & 11 & 190 & 397 & 0 & 84 & 90 & 93 & $* 95$ & 92 & 91 & 40 & 64 & 78 & 38 & 62 & 56 \\
\hline hyp ×hyp & 3 & 110 & 224 & 11 & 7 & 278 & 630 & 1 & 81 & 78 & 96 & 96 & 92 & 89 & 13 & -59 & 89 & 10 & 44 & 19 \\
\hline Davison \& Hunter & 3 & 95 & 252 & 8 & 19 & 371 & 745 & 1 & 82 & 73 & 96 & 94 & 91 & 87 & 0 & -167 & 92 & 45 & 49 & 4 \\
\hline Stevens & 2 & 77 & 245 & 83 & 3 & 454 & 863 & 1 & 82 & 72 & 90 & *95 & 90 & 86 & 0 & -190 & 94 & 37 & 33 & -5 \\
\hline Herrnstein & 2 & 146 & 489 & 37 & 28 & 628 & 1327 & 0 & 77 & 3 & 94 & 93 & 88 & 71 & -63 & -11 & 67 & -140 & 74 & -15 \\
\hline \multicolumn{21}{|c|}{ Modeling of residence time or time spent per min } \\
\hline Model & $m$ & $D M$ & Mur & $\mathrm{DH}$ & $P B$ & $E D$ & Sum & CSet & $D M$ & Mur & $\mathrm{DH}$ & $P B$ & $E D$ & Mean & $D M$ & Mur & $\mathrm{DH}$ & $P B$ & $E D \nLeftarrow$ & Mean \\
\hline pow $\times$ hypp + b & 5 & 0 & 1 & & 12 & 8 & 21 & 3 & *93 & *95 & - & $* 95$ & $* 53$ & 84 & 98 & 94 & - & 59 & 91 & 85 \\
\hline hyp $\times$ hypp + b & 5 & 3 & 0 & & 11 & 19 & 33 & 2 & $* 93$ & *95 & - & *95 & *54 & 84 & 98 & 94 & - & 37 & 91 & 80 \\
\hline pow $x$ hyp + b & 4 & 77 & 70 & & 7 & 0 & 154 & 2 & 89 & 90 & - & *95 & *53 & 82 & 79 & 87 & - & 46 & 91 & 76 \\
\hline hypxhyp + b & 4 & 112 & 78 & & 6 & 4 & 201 & 2 & 89 & 90 & - & *95 & *54 & 82 & 72 & 83 & - & 18 & 91 & 66 \\
\hline pow $\times$ hyp & 3 & 79 & 44 & & 0 & 312 & 434 & 1 & 89 & 91 & - & *95 & 26 & 75 & 81 & 85 & - & 52 & 82 & 75 \\
\hline hypxhyp & 3 & 118 & 64 & & 19 & 312 & 513 & 0 & 87 & 89 & - & $* 94$ & 26 & 74 & 70 & 77 & - & -248 & 82 & -5 \\
\hline Davison \& Hunter & 3 & 335 & 367 & & 118 & 452 & 1273 & 0 & 67 & 48 & - & 75 & 20 & 52 & -3 & -85 & - & -594 & 73 & -152 \\
\hline Stevens & 2 & 372 & 384 & & 141 & 463 & 1360 & 0 & 60 & 38 & - & 67 & 16 & 45 & -3 & -106 & - & -753 & 71 & -198 \\
\hline Herrnstein & 2 & 451 & 737 & & 274 & 670 & 2131 & 0 & 42 & -340 & - & -81 & 10 & -92 & -94 & -130 & - & -348 & 84 & -122 \\
\hline
\end{tabular}

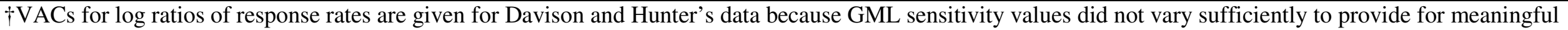

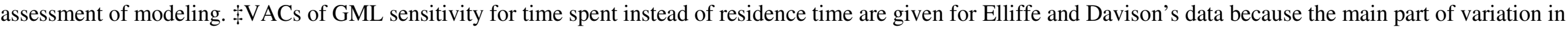
time allocation was attributed to the differences in changeover rate to different alternatives and not to the residence time itself 
Table 2

Rank of the models consistent with generalized matching principle against 20 Navakatikyan's models. Ranks are in increasing order of $\triangle \mathrm{AICc}$ values. Abbreviations of the experiments are as in Table 1 . CO is modelling of changeover rate. Models are described in Table A1, Appendix A. The model listed here as 'Davison $\&$ Hunter' is Equation 5 of the current paper.

\begin{tabular}{|c|c|c|c|c|c|c|c|c|c|c|}
\hline \multirow[t]{2}{*}{ Model } & \multicolumn{5}{|c|}{ Response rate modelling } & \multicolumn{4}{|c|}{ Residence Time modelling } & $\mathrm{CO}$ \\
\hline & $* D M$ & Mur & $* * D H$ & $* * P B$ & $E D$ & $D M$ & Mur & $P B$ & $E D$ & $E D$ \\
\hline Davison \& Hunter & 21 & 22 & 14 & 17 & 21 & 21 & 21 & 21 & 21 & 21 \\
\hline Davison \& Hunter B & 20 & 23 & 5 & 15 & 22 & 22 & 23 & 23 & 23 & 22 \\
\hline Stevens & 18 & 21 & 24 & 9 & 23 & 23 & 22 & 22 & 22 & 23 \\
\hline Herrnstein & 24 & 24 & 17 & 22 & 24 & 24 & 24 & 24 & 24 & 24 \\
\hline
\end{tabular}

* Two simple Navakatikyan's models performed worse than the competitors

** Models performed on par with Navakatikyan's models 


\section{FIGURE CAPTIONS}

Fig. 1. Predictions of the component-functions model for interdependent concurrent VI VI performance (Navakatikyan, 2007) without bias. A. Change in sensitivity parameter $a$ calculated over 5 relative reinforcer rates $(0.1,0.2,0.5,0.8,0.9)$ for each of 6 overall reinforcer rates $(0.2,0.5,1,2,5$, and $10 \mathrm{rfts} / \mathrm{min}$ obtained $)$ for the arithmetic and exponential schedule. B. Log response ratios vs. log reinforcer ratios, and the respective point sensitivities calculated as $\log \left(T_{1} / T_{2}\right) / \log \left(R_{1} / R_{2}\right)$ according to the residence times values from the unbiased model of Bird 136 for the arithmetic schedule. C. Three-alternative extension of the model. Prediction for sensitivity parameter $a$ for two alternatives while relative reinforcer rate on the 3rd alternative is increasing. Overall reinforcer rate obtained was set to $2 \mathrm{rfts} / \mathrm{min}$, ratio $R_{1} / R_{2}=1 / 6$. The data are from 6 pigeons responding on arithmetic (Alsop \& Elliffe, 1988) and exponential (Elliffe \& Alsop, 1996) VI VI schedules. The calculations were made as part of the present paper.

Fig. 2. Model fits for interdependent concurrent VI VI VI schedules with random-switching procedure. Behavior is ordered in increasing order of arranged reinforcer rates per minute for each condition and plotted against reinforcer rate of "third" alternative. In the upper left panel the arranged rates for the "first" and "second" alternatives are presented and should be applied across all panels. Models are based on Herrnstein's, Davison and Hunter's and the best Navakatikyan's (power×hyperbolic-power function with baseline constant, Equation 13) LOE equations. Data are from Bird 104 of Davison and McCarthy's (1994) Part B experiment.

Fig. 3. Prediction of generalized-matching sensitivities for interdependent concurrent VI VI VI schedules with random-switching procedure. Low and high denote the low- and high-other-reinforcer-rate groups (see text for explanation). Models' predictions are connected by lines to aid visual perception. Models are based on Herrnstein's, Davison and Hunter's and the best Navakatikyan's (power×hyperbolic-power function with baseline constant, Equation 13) LOE equations. Data are from Davison and McCarthy's (1994) Part B experiment.

Fig. 4. Model fits for interdependent concurrent VI VI VI schedules with random-switching procedure and increasing relative reinforcer rate on the third alternative. Overall arranged reinforcer rate is 1.332 reinforcers per minute; arranged ratio $R_{1}: R_{2}=6: 1$. Only half of the data, i.e. the conditions in which the first alternative was richer are presented; the conditions with arranged ratio $R_{1}: R_{2}=1: 6$ are omitted. Models are based on Herrnstein's, Davison and Hunter's and the best Navakatikyan's (power-hyperbolic function with baseline constant, Equation 11, for response rate; and hyperbolicxhyperbolic-power function with baseline constant, Equation 12, for residence time) LOE equations. Data are from Bird 44 of Murrell's (1995) Part B experiment. 
Fig. 5. Prediction of generalized matching sensitivities for interdependent concurrent VI VI VI schedules with random-switching procedure and increasing relative reinforcer rate on the third alternative. Models are based on Herrnstein's, Davison and Hunter's and the best Navakatikyan's (power-hyperbolic function with baseline constant, Equation 11, for response rate; and hyperbolicxhyperbolic-power function with baseline constant, Equation 12, for residence time) LOE equations. Data are from Murrell's (1995) Part B experiment.

Fig. 6. Model fits for independent concurrent VI VI VI schedules arranged on three, two and one key(s). Data averaged over subjects are from Davison and Hunter (1976). In titles, X denotes varying VI schedule, EXT denotes extinction. Left three panels are for Set 1 and 2 conditions with varying VI schedule (top), VI $120 \mathrm{sec}$ (middle), and VI $60 \mathrm{~s}$ (bottom). Middle three panels are for Set 3 and 4 conditions with varying VI schedule (top), VI $120 \mathrm{sec}$ (middle), and extinction schedule (bottom). Right three panels are for Set 5 conditions with varying VI schedule (top) and extinction schedules (middle and bottom). The bottom-most graph gives non-extinction reinforcer rates for the constant VI schedules for Sets 1 and 2, 3 and 4. Models are based on Herrnstein's, Davison and Hunter's and the best Navakatikyan's (hyperbolic xhyperbolic function with baseline constant, Equation 9) LOE equations.

Fig. 7. Prediction of generalized matching sensitivities for independent concurrent VI VI VI schedules arranged on three, two and one key(s). Data averaged over subjects are from Davison and Hunter (1976).

Sensitivity is calculated for 6 subsets of the data as described in the main text. Models' predictions are presented as lines to aid visual perception. Models are based on Herrnstein's, Davison and Hunter's, the best Navakatikyan's (hyperbolicxhyperbolic function with baseline constant, Equation 9) LOE equations.

Fig. 8. Model fits for independent concurrent VI VI VI schedules in switching-key procedure. Data are from Bird 7, Pliskoff and Brown (1976). Data are re-ordered by conditions, with the largest arranged reinforcer rate assigned to be the first alternative, the second largest to the second alternative, and the smallest arranged reinforcer rate to the third alternative. Data are plotted against the reinforcer rate on the richest alternative. Models are based on Herrnstein's, Davison and Hunter's, and the best Navakatikyan's (hyperbolicxhyperbolic-power function with baseline constant, Equation 12, for response rate; and powerxhyperbolic function, Equation 10, for residence time) LOE models. The bottom-most graph gives obtained reinforcer rates for other alternatives plotted against the richest alternative.

Fig. 9. Prediction of generalized matching sensitivities for independent concurrent VI VI VI schedules in switching-key procedure. Data are from Pliskoff and Brown (1976). Sensitivity is calculated for 3 subsets of the data: between 1st and 2nd, 1st and 3rd, and 2nd and 3rd alternatives. Models' predictions are presented as lines to 
aid visual perception. Models are based on Herrnstein's, Davison and Hunter's, and the best Navakatikyan's (hyperbolicxhyperbolic-power function with baseline constant, Equation 12, for response rate; and powerxhyperbolic function, Equation 10, for residence time) LOE models. Predictions for sensitivity for Herrnstein's model are always equal to 1 for all birds (solid line).

Fig. 10. Model fits for the response rate on the four-key concurrent VI VI VI VI rapid-change procedure. The panels from the top to the bottom are showing performance in the alternatives from the higher to the lower probability of reinforcement. Each panel represents four graphs for the four different previous/preceding schedules. Models are based on Herrnstein's, Davison and Hunter's, and the best Navakatikyan's (hyperbolicxhyperbolicpower function with baseline constant, Equation 12) LOE models. Data are from Bird 85, Elliffe and Davison (2010).

Fig. 11. Model fits averaged over birds and the previous conditions for the four-key concurrent VI VI VI VI rapid-change procedure. Probabilities of reinforcement over 40 parts are denoted as 27, 9, 3 and 1; in brackets, there are VI schedule parameters related to the probabilities. Overall reinforcer rate arranged at 3 reinforcers per minute. Point for the first reinforcer is the same for data and models, serving as the starting state for the dynamical modeling; the point is not counted in VAC assessment. In the left top corner of panels is VAC for the model. Models are based on Herrnstein's, Davison and Hunter's, and the best Navakatikyan's (hyperbolicxhyperbolicpower function with baseline constant, Equation 12, for the response rate and changeover rate; and powerxhyperbolic function with baseline constant, Equation 11, for residence time) LOE models. Data are from Elliffe and Davison (2010).

Fig. 12. Dynamics of the generalized point-sensitivities in the four-key concurrent VI VI VI VI rapid-change procedure. Curves and symbols represent the sensitivities between alternatives with probabilities of reinforcer 27 to 9 (27:9), 9 to 3 (9:3) and 3 to 1(3:1) over 40 reinforcers delivered. The models and averaging procedure were the same as in Fig. 11. Data are from Elliffe and Davison (2010). 
A. INCREASING OVERALL REINFORCER RATE

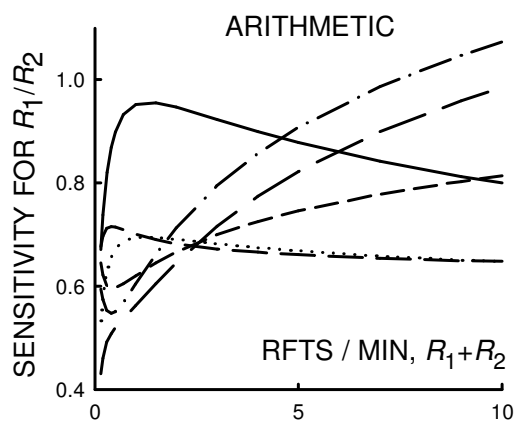

EXPONENTIAL

B. DIFFERENT RELATIVE AND OVERALL REINFORCER RATES
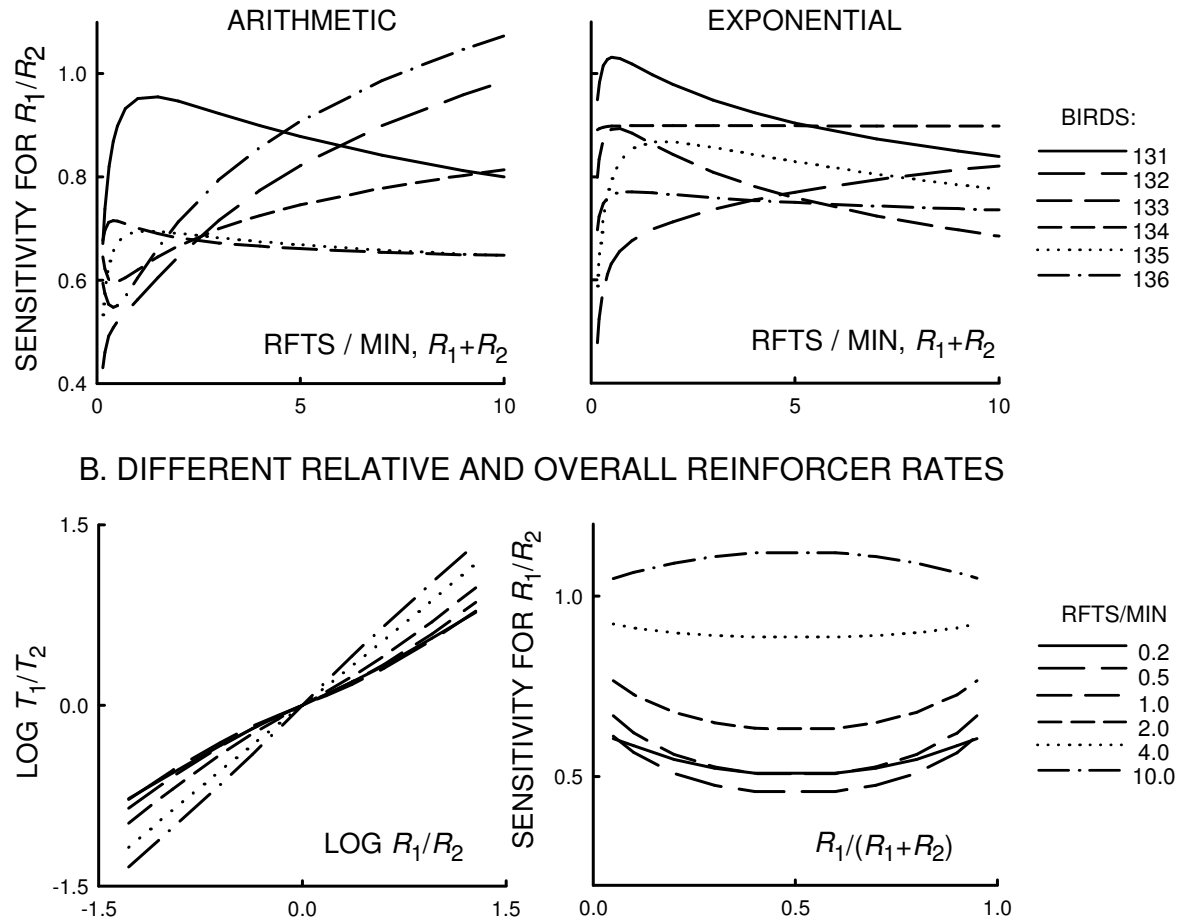

LOG $R_{1} / R_{2}$

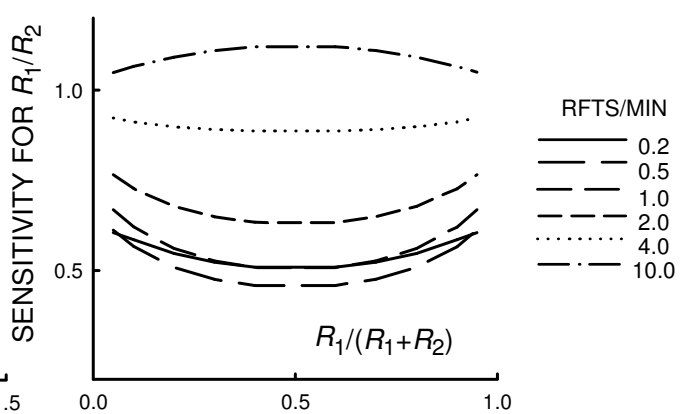

C. INCREASING RELATIVE REINFORCER RATE ON 3RD ALTERNATIVE
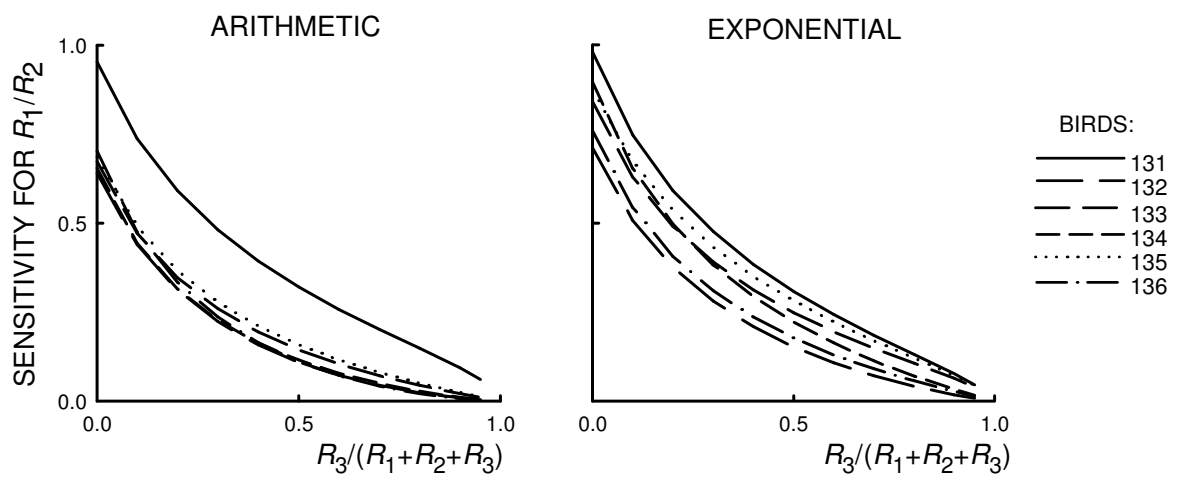

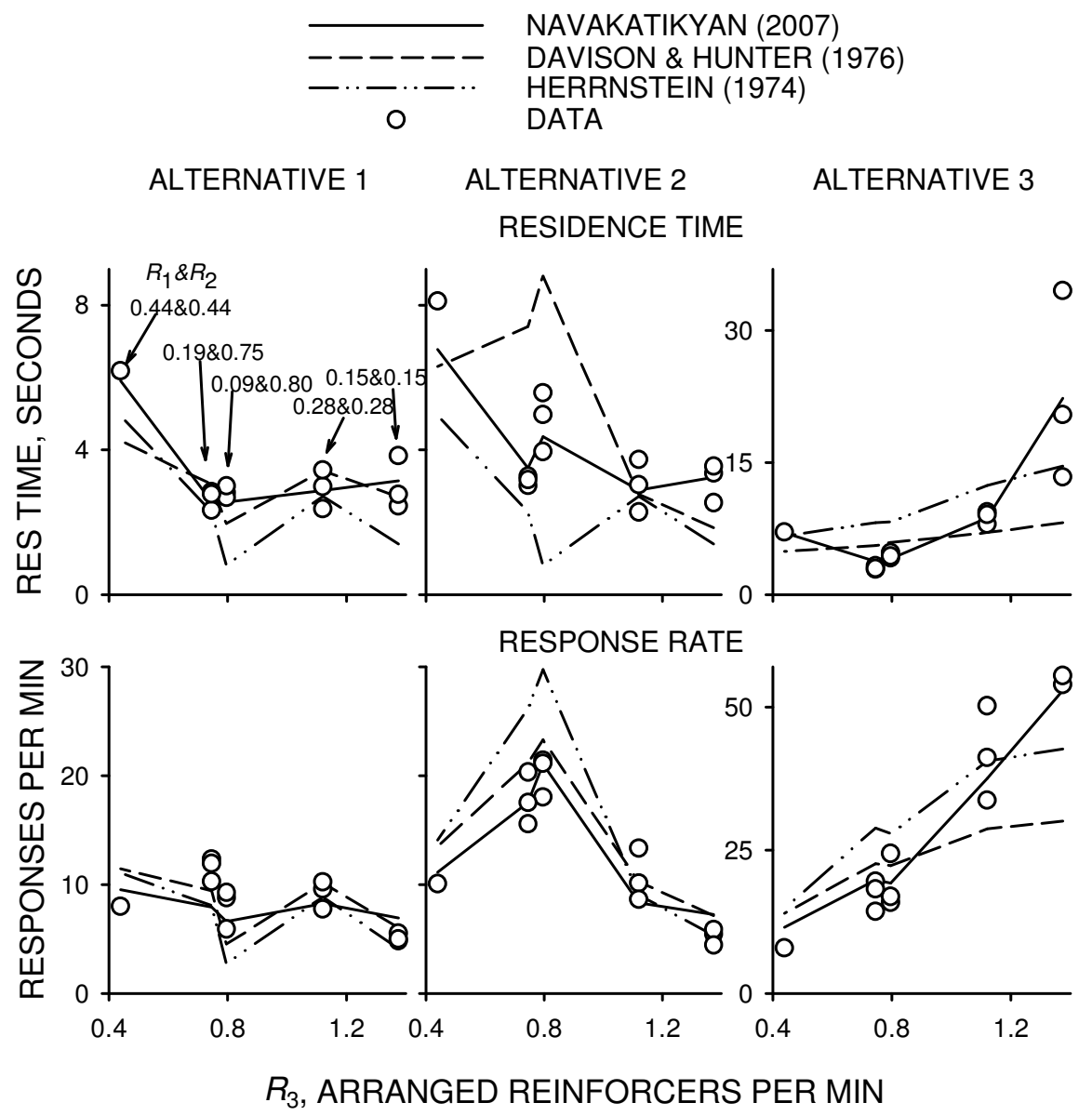


$$
\begin{aligned}
& \text { - } .+\cdots \cdot \text { NAVAKATIKYAN, LOW } \\
& \text {-..-.... NAVAKATIKYAN, HIGH } \\
& \text { - - - - - DAVISON \& HUNTER, LOW \& HIGH } \\
& \text { HERRNSTEIN, LOW \& HIGH } \\
& \square \quad \text { DATA, LOW } \\
& \text { D DATA, HIGH }
\end{aligned}
$$

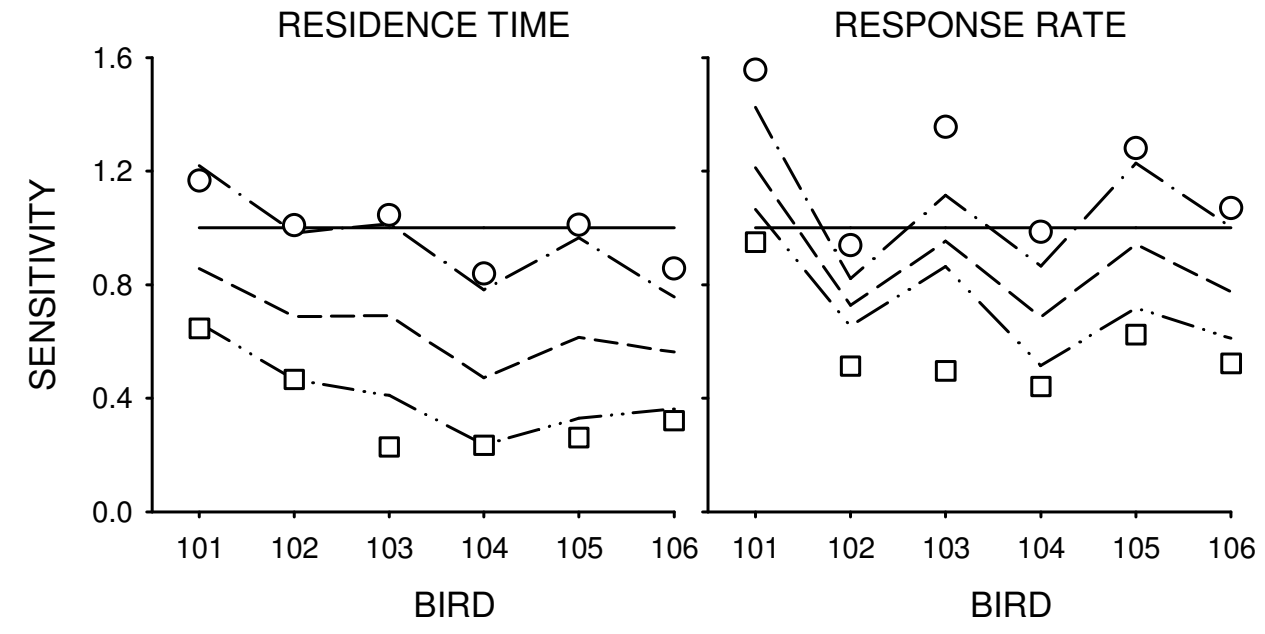



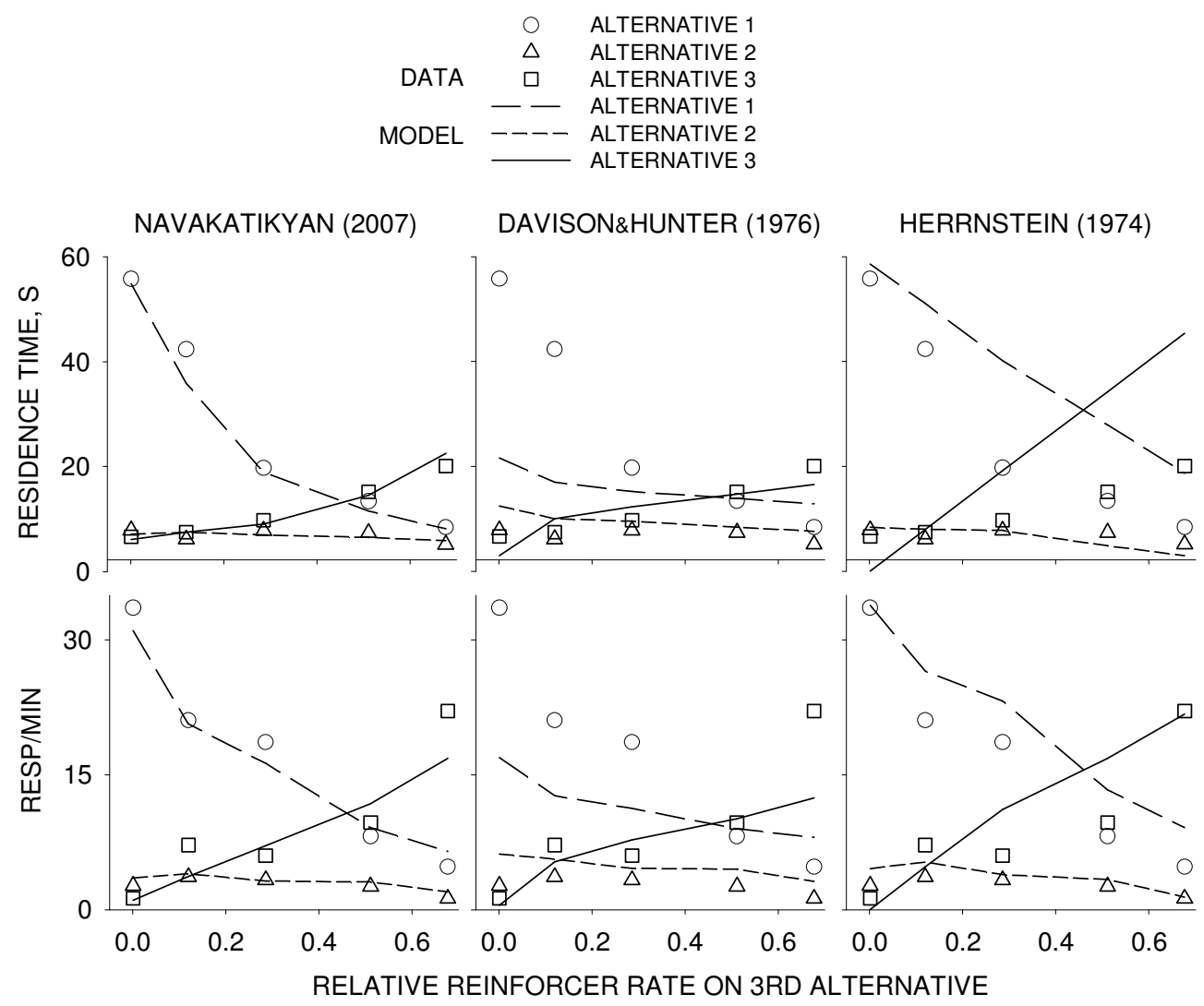


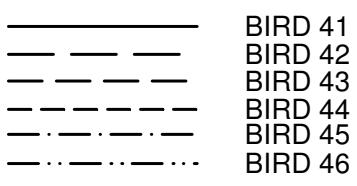

DATA

NAVAKATIKYAN DAVISON \& HUNTER

(2007) HERRNSTEIN

RESIDENCE TIME

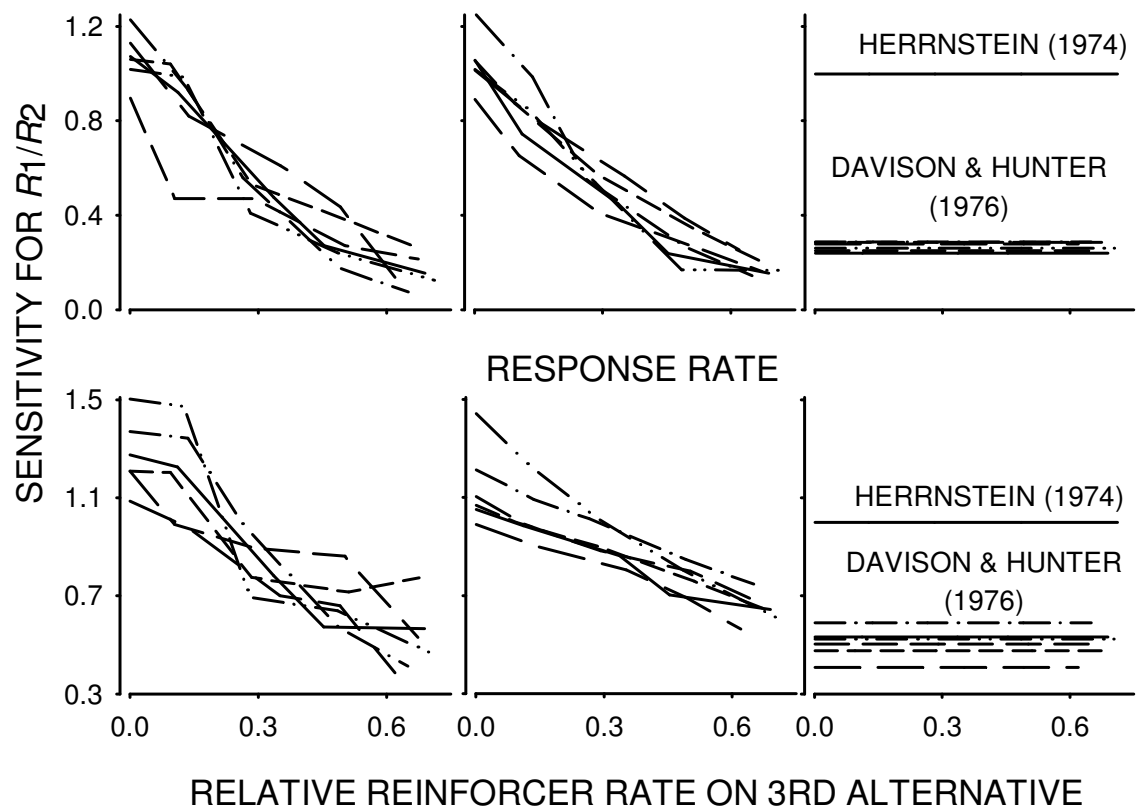




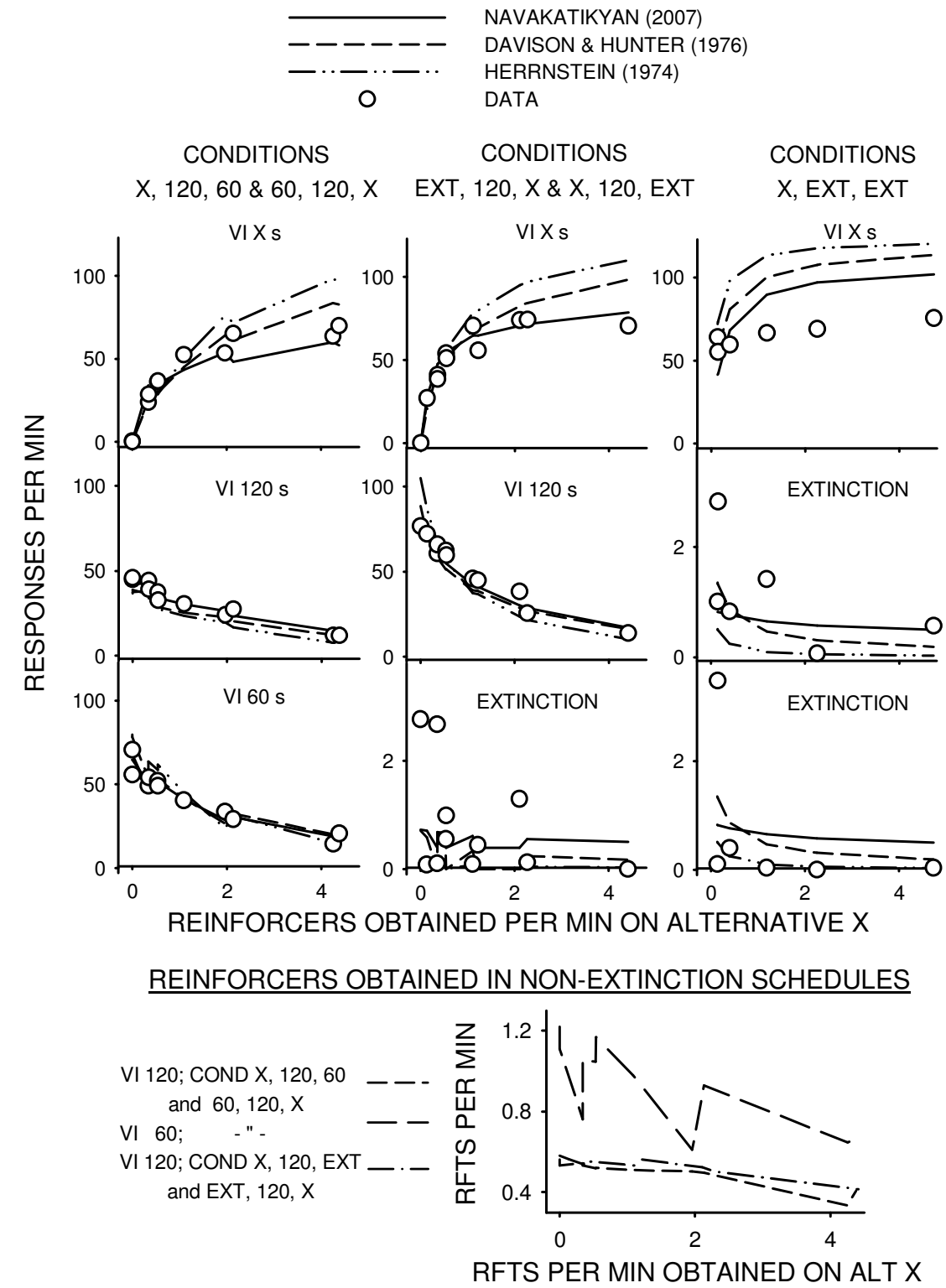




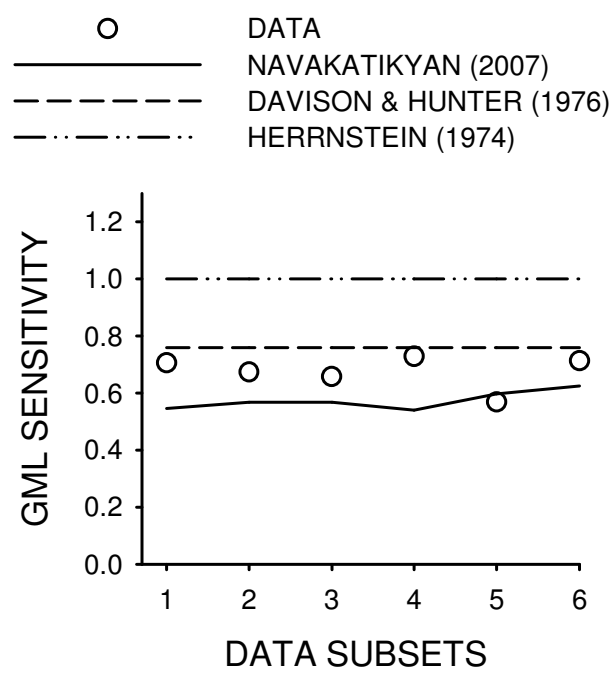




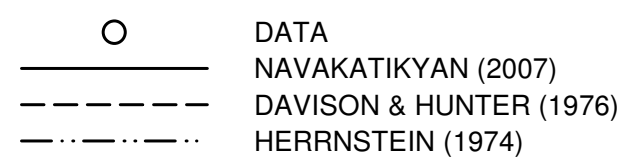

ALTERNATIVE 1

(THE RICHEST)
ALTERNATIVE 2 (THE MIDDLE)

RESIDENCE TIME
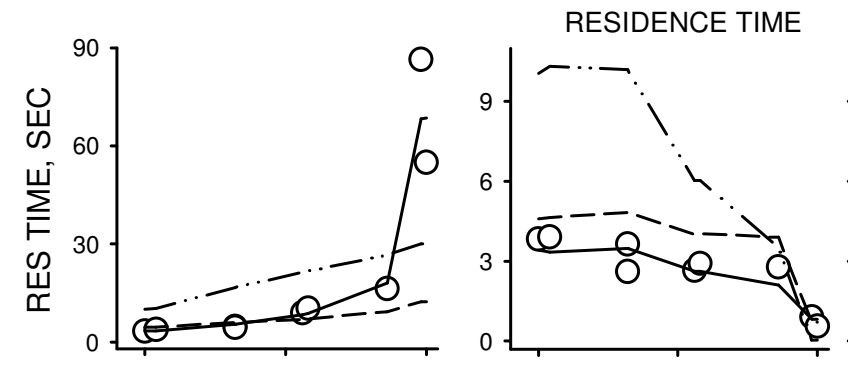

ALTERNATIVE 3

(THE POOREST)
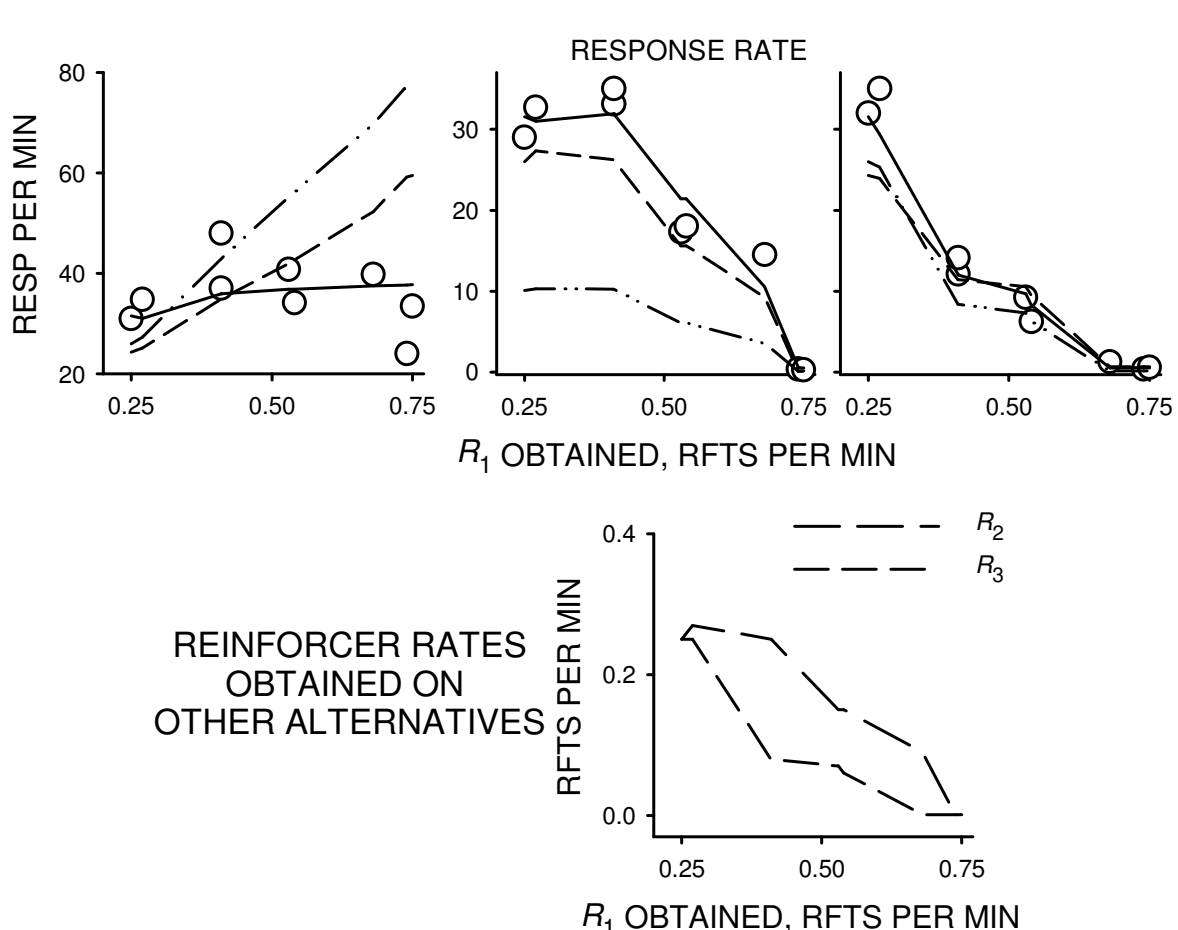


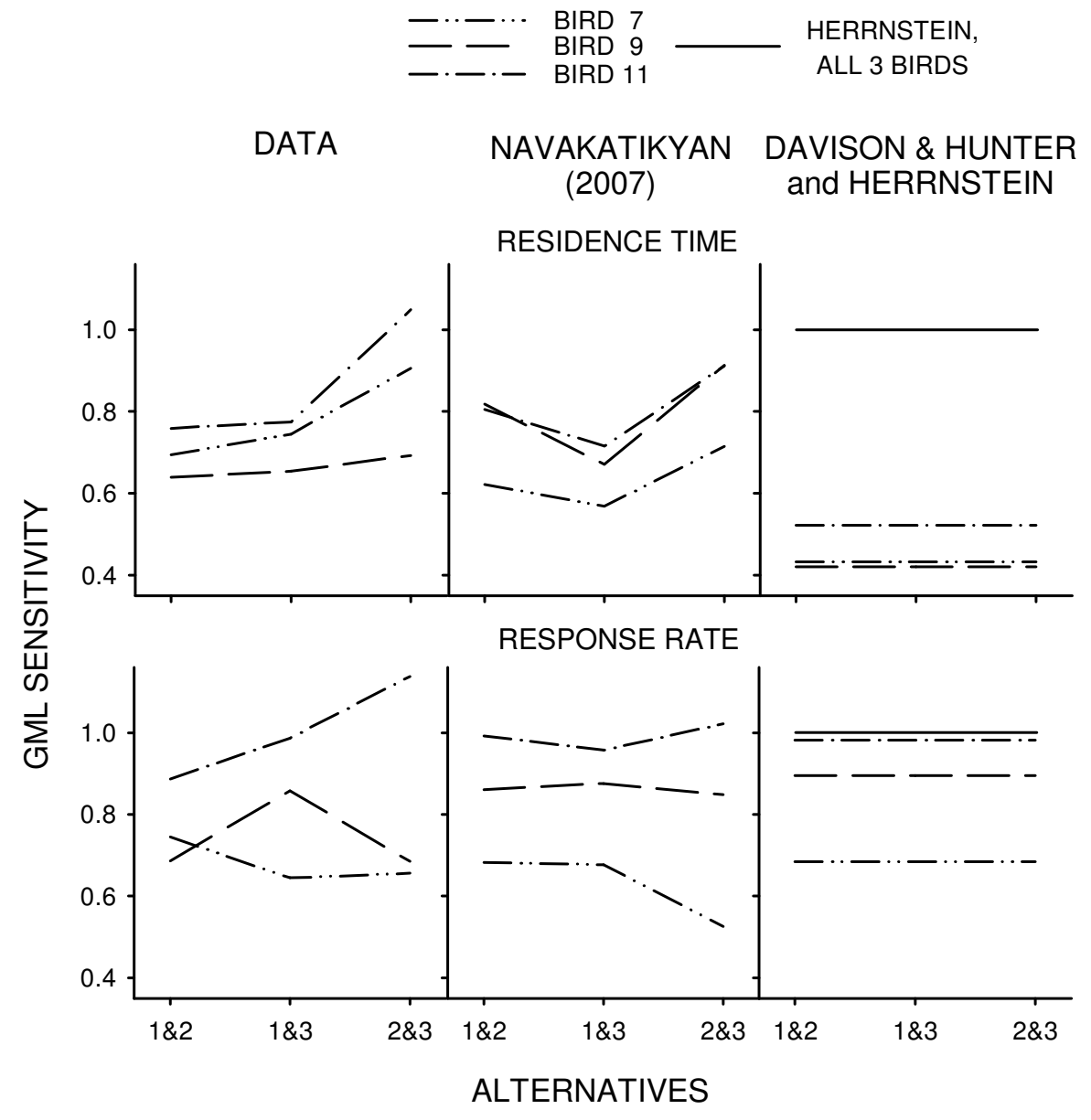




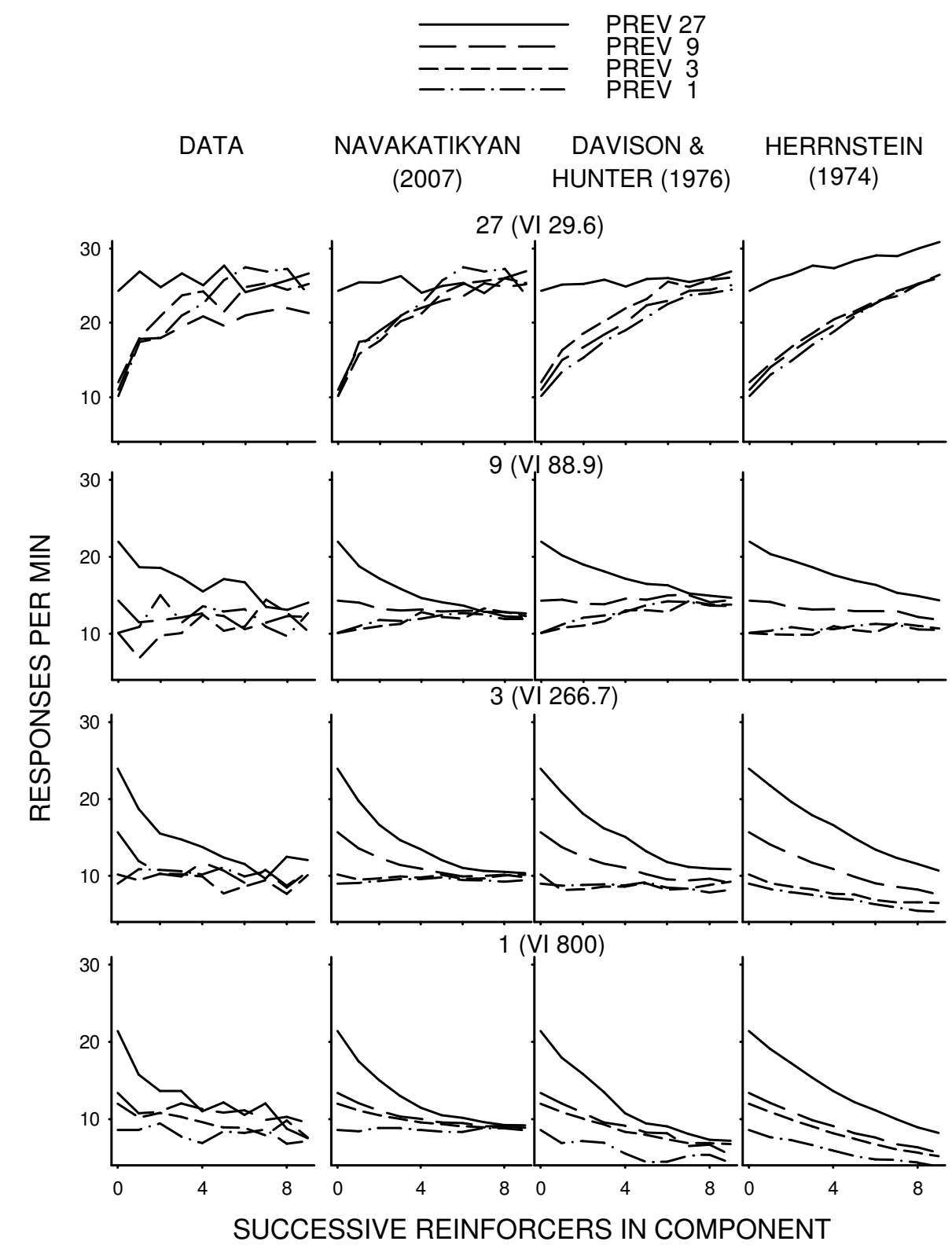




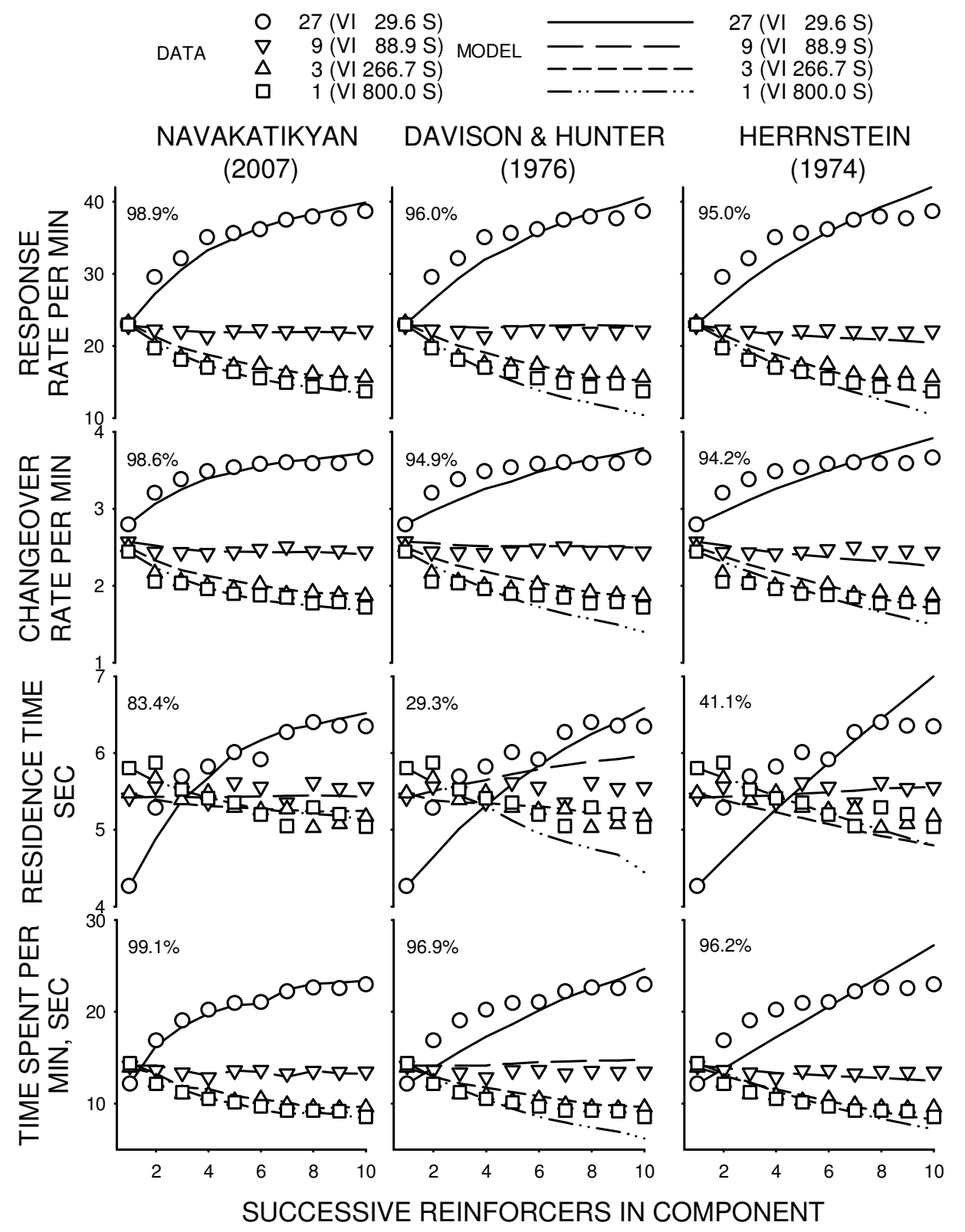




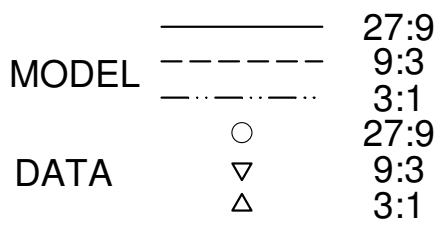

NAVAKATIKYAN (2007)

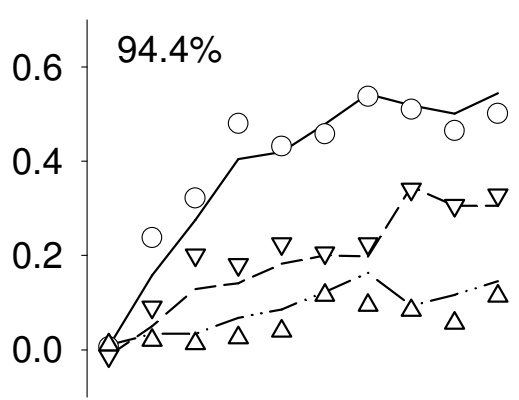

$88.1 \%$

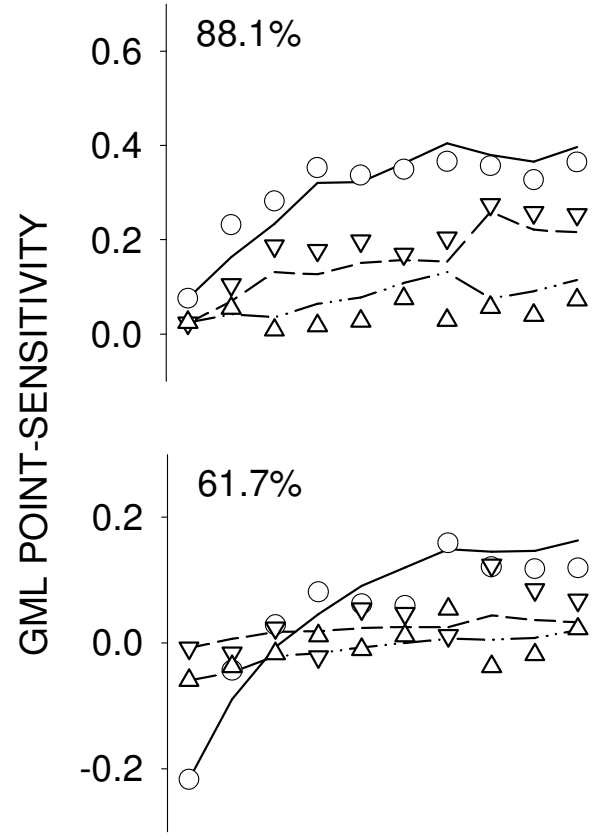

TIME PER MINUTE SPENT ON ALTERNATIVE

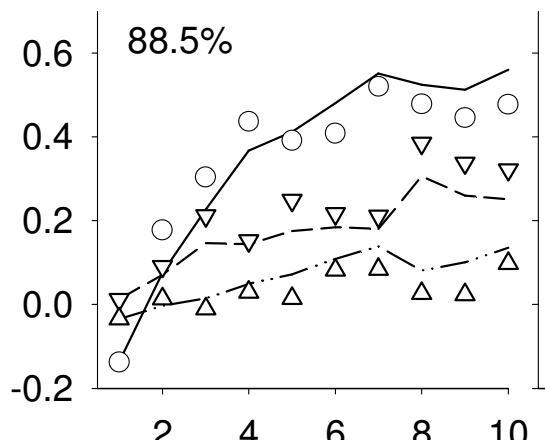

$34.6 \%$

\section{RESIDENCE TIME} $-61.7 \%$

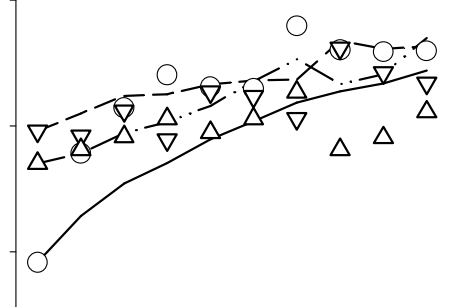

$10.1 \%$

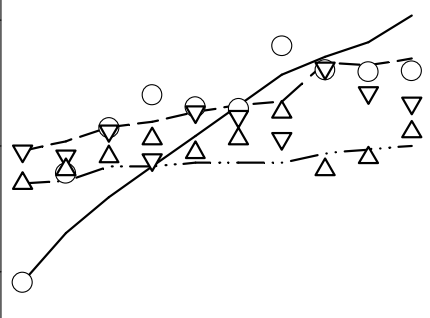
$67.9 \%$

\section{CHANGEOVER RATE}

$74.5 \%$

(1974)
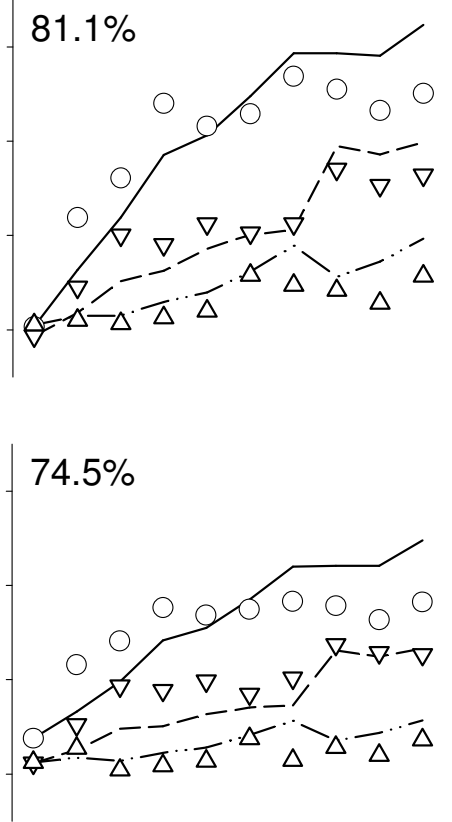


\section{APPENDIX A}

\section{Full Set OF AsSESSED Models}

Table A1

Models of choice consistent with the matching principle. Abbreviations: $m$ is number of adjustable parameters in a function; $R, R_{\text {oth }}$ are reinforcer rates on current alternative and sum of reinforcer rates on other alternatives; $R_{\text {othl }}$, $R_{\text {oth } 2}$ are reinforcer rates on $1^{\text {st }}, 2^{\text {nd }}$, etc. other alternatives. Constants of the models are denoted as $a, k, s$. Models included in the main subset for the detailed description in the current article are flagged with dagger $(\dagger)$.

\begin{tabular}{|r|l|l|l|}
\hline No & Model equation & $m_{\mathrm{t}}$ & Description \\
\hline$\dagger 1$ & $B=a R^{k}$ & 2 & Stevens \\
\hline$\dagger 2$ & $B=a R /\left(R+R_{\text {oth }}+k\right)$ & 2 & Herrnstein \\
\hline 3 & $B=a\left[R /\left(R+R_{\text {oth }}+k\right)\right]^{s}$ & 3 & Davison \& Hunter B \\
\hline$\dagger 4$ & $B=a R^{s} /\left(R^{s}+\left(R_{\text {othl }}\right)^{s}+\left(R_{\text {oth } 2}\right)^{s}+\ldots+k\right)$ & 3 & Davison \& Hunter \\
\hline
\end{tabular}


Table A2

Enhancing and reducing component functions used for modeling choice. Abbreviations: $F_{\text {enh }}$ and $F_{\text {red }}$ are enhancing and reducing component-functions. Constants of the models are denoted as $a, k, k_{r e d}, s . R, R_{o t h}$ are reinforcers on current alternative and sum of reinforcers on other alternatives; exp is an exponential function. Other abbreviations are as in Table A1.

\begin{tabular}{|l|l|l|l|l|}
\hline No & Equations & $m$ & Description of function & Abbreviation \\
\hline \multicolumn{2}{|c|}{ Enhancing component-functions } \\
\hline 1 & $F_{\text {enh }}=a R /(R+k)$ & 2 & Hyperbolic & hyp \\
\hline 2 & $F_{\text {enh }}=a R^{k}$ & 2 & Power & pow \\
\hline 3 & $F_{\text {enh }}=a[1-\exp (-k R)]$ & 2 & Exponential, bounded, 2 parameters & exp \\
\hline 4 & $F_{\text {enh }}=a+c R /(R+k)$ & 3 & Hyperbolic plus constant & ahyp \\
\hline 5 & $F_{\text {enh }}=a+b R^{k}$ & 3 & Power plus constant & apow \\
\hline 6 & $F_{\text {enh }}=[(a-c) \cdot \exp (-k R)+c]$ & 3 & Exponential, bounded, 3 parameters & aexp \\
\hline 7 & $F_{\text {enh }}=c /[1+(c / a-1) \cdot \exp (-k R)]$ & 3 & Logistic & logt \\
\hline 8 & $F_{\text {enh }}=a\left\{1-\exp \left[-(k R)^{s}\right]\right\}$ & 3 & Exponential-power & expp \\
\hline \multicolumn{7}{|l|}{ Reducing component-functions } \\
\hline 1 & $F_{\text {red }}=1 /\left(1+R_{\text {oth }} / k_{\text {red }}\right)$ & 1 & Hyperbolic & hyp \\
\hline 2 & $F_{\text {red }}=1 /\left[1+\left(R_{\text {oth }} / k_{\text {red }}\right)^{s}\right]$ & 2 & Hyperbolic-power & hypp \\
\hline 3 & $F_{\text {red }}=\exp \left\{-\left[\left(R_{\text {oth }} / k_{\text {red }}\right)^{s}\right]\right\}$ & 2 & Exponential-power & dlogt \\
\hline 4 & $F_{\text {red }}=d+1-1 /\left[1+(1 / d-1) \cdot \exp \left(-R_{\text {oth }} / k_{\text {red }}\right)\right]$ & 2 & Decreasing logistic \\
\hline
\end{tabular}


Table A3

Component-functions models of choice. Number of parameters in component functions and overall in the model are denoted as $m_{\mathrm{enh}}, m_{\mathrm{red}}, m_{\mathrm{tot}}$, respectively. Baseline constant is denoted as $\mathrm{b}$. The dynamical models have one extra parameter - dynamical constant $k_{t}$. Other abbreviations are as in Table A1.

\begin{tabular}{|c|c|c|c|c|c|}
\hline No & Model equation in the form of $F_{\text {enh }} \times F_{\text {red }}+b$ & $m_{\mathrm{enh}}$ & $m_{\text {red }}$ & $m_{\text {tot }}$ & Description \\
\hline$\dagger 1$ & $\times\left[1 /\left(1+R_{\text {oth }} / k_{\text {red }}\right)\right]$ & 2 & 1 & 3 & hyp xhyp \\
\hline$\dagger 2$ & $\times\left[1 /\left(1+R_{\text {oth }} / k_{\text {red }}\right)\right]$ & 2 & 1 & 3 & pow $\times$ hyp \\
\hline 3 & $\times\left[1 /\left(1+R_{\text {oth }} / k_{\text {red }}\right)\right]$ & 2 & 1 & 3 & exp $\times$ hyp \\
\hline$\uparrow 4$ & $\times\left[1 /\left(1+R_{\text {oth }} / k_{\text {red }}\right)\right]+b$ & 2 & 1 & 4 & hyp $\times$ hyp + b \\
\hline$\dagger 5$ & $\times\left[1 /\left(1+R_{\text {oth }} / k_{\text {red }}\right)\right]+b$ & 2 & 1 & 4 & pow $\times$ hyp $+b$ \\
\hline 6 & $\times\left[1 /\left(1+R_{\text {oth }} / k_{\text {red }}\right)\right]+b$ & 2 & 1 & 4 & exp $\times h y p+b$ \\
\hline 7 & $\times\left[1 /\left(1+R_{\text {oth }} / k_{\text {red }}\right)\right]+b$ & 3 & 1 & 5 & ahyp $\times$ hyp + b \\
\hline 8 & $\times\left[1 /\left(1+R_{\text {oth }} / k_{\text {red }}\right)\right]+b$ & 3 & 1 & 5 & apow $\times$ hyp $+b$ \\
\hline 9 & $\times\left[1 /\left(1+R_{\text {oth }} / k_{\text {red }}\right)\right]+b$ & 3 & 1 & 5 & aexp $\times$ hyp $+b$ \\
\hline 10 & $B=\{c /[1+(c / a-1) \cdot \exp (-k R)]\} \times\left[1 /\left(1+R_{\text {oth }} / k_{\text {red }}\right)\right]+b$ & 3 & 1 & 5 & $\operatorname{logt} \times$ hyp $+b$ \\
\hline 11 & $\times\left[1 /\left(1+R_{\text {oth }} / k_{\text {red }}\right)\right]+b$ & 3 & 1 & 5 & expp $\times$ hyp $+b$ \\
\hline$\dagger 12$ & $\times\left\{1 /\left[1+\left(R_{o t h} / k_{r e d}\right)^{s}\right]\right\}+b$ & 2 & 2 & 5 & hyp $\times$ hypp + b \\
\hline$\dagger 13$ & $\times\left\{1 /\left[1+\left(R_{\text {oth }} / k_{\text {red }}\right)^{s}\right]\right\}+b$ & 2 & 2 & 5 & pow $\times$ hypp + b \\
\hline 14 & $\times\left\{1 /\left[1+\left(R_{\text {oth }} / k_{\text {red }}\right)^{s}\right]\right\}+b$ & 2 & 2 & 5 & exp $\times h y p p+b$ \\
\hline 15 & $\times \exp \left\{-\left[\left(R_{\text {oth }} / k_{\text {red }}\right)^{s}\right]\right\}+b$ & 2 & 2 & 5 & hyp $\times \operatorname{expp}+b$ \\
\hline 16 & $\times \exp \left\{-\left[\left(R_{\text {oth }} / k_{\text {red }}\right)^{s}\right]\right\}+b$ & 2 & 2 & 5 & pow $\times \operatorname{expp}+b$ \\
\hline 17 & $\times \exp \left\{-\left[\left(R_{\text {oth }} / k_{\text {red }}\right)^{s}\right]\right\}+b$ & 2 & 2 & 5 & $\exp \times \operatorname{expp}+b$ \\
\hline 18 & $\times\left\{d+1-1 /\left[1+(1 / d-1) \cdot \exp \left(-R_{\text {oth }} / k_{\text {red }}\right)\right]\right\}+b$ & 2 & 2 & 5 & hyp $\times d \log t+b$ \\
\hline 19 & $\times\left\{d+1-1 /\left[1+(1 / d-1) \cdot \exp \left(-R_{\text {ott }} / k_{\text {red }}\right)\right]\right\}+b$ & 2 & 2 & 5 & pow $\times d \log t+b$ \\
\hline 20 & $B=a[1-\exp (-k R)] \times\left\{d+1-1 /\left[1+(1 / d-1) \cdot \exp \left(-R_{\text {oth }} / k_{\text {red }}\right)\right]\right\}+b$ & 2 & 2 & 5 & $\exp \times d \log t+b$ \\
\hline
\end{tabular}


Table A4

Comparison of the performance of the full set of models we considered. DM, Mur, DH, PB, ED refer to Davison and McCarthy's (1994), Murrell's (1995), Davison and Hunter's (1976), Pliskoff and Brown's (1976), and Elliffe and Davison's (2010) data. The measure is $\Delta$ AICc, the difference of each model's second-order Akaike information criterion from that of the best model. The differences are recalculated for the full set of models and thus differ from the ones derived for the subset of models considered in the article in detail (flagged with dagger, $\dagger$ ). $m$ is the number of parameters in each model. The models are ordered by the sum of $\Delta$ AICc across experiments. Abbreviations for the models are as in Table A2 and A3. Note that the values of $\triangle \mathrm{AICc}$ in this table could be different from the values in Table 1, because the minimal AICc values used for the calculation of $\triangle \mathrm{AICc}$ are in some cases different for the full set of models (this table) and for the subset of the models selected for detailed representation (Table 1).

\begin{tabular}{|c|c|c|c|c|c|c|c|c|c|c|c|c|c|c|}
\hline \multicolumn{8}{|c|}{ Modeling of response rate } & \multicolumn{7}{|c|}{ Modeling of residence time } \\
\hline \multirow{2}{*}{ Model } & \multirow{2}{*}{$m$} & \multicolumn{5}{|c|}{ Data } & \multirow{2}{*}{ Sum } & \multirow{2}{*}{ Model } & \multirow{2}{*}{$m$} & \multicolumn{4}{|c|}{ Data } & \multirow{2}{*}{ Sum } \\
\hline & & $D M$ & Mur & $\mathrm{DH}$ & $P B$ & $E D$ & & & & $D M$ & Mur & $P B$ & $E D$ & \\
\hline $\exp \times d \log t+b$ & 5 & 18 & 10 & 10 & 2 & 4 & 44 & pow $\times$ hypp $+b$ & 5 & 1 & 1 & 12 & 18 & 32 \\
\hline expp $\times$ hyp $+b$ & 5 & 18 & 24 & 8 & 4 & 0 & 54 & pow $\times \operatorname{expp}+b$ & 5 & 0 & 9 & 14 & 17 & 40 \\
\hline hyp $\times d \log t+b$ & 5 & 32 & 12 & 8 & 5 & 14 & 71 & hyp $\times$ hypp $+b$ & 5 & 5 & 0 & 11 & 28 & 44 \\
\hline exp $\times$ hypp $+b$ & 5 & 42 & 26 & 7 & 0 & 18 & 93 & exp $\times$ hypp $+b$ & 5 & 2 & 4 & 12 & 27 & 45 \\
\hline exp $\times h y p+b$ & 4 & 59 & 11 & 6 & 7 & 14 & 97 & $\exp \times \operatorname{expp}+b$ & 5 & 1 & 10 & 11 & 29 & 51 \\
\hline $\exp \times \operatorname{expp}+b$ & 5 & 48 & 31 & 6 & 1 & 13 & 99 & hyp $\times \operatorname{expp}+b$ & 5 & 4 & 10 & 11 & 27 & 52 \\
\hline pow $\times d \log t+b$ & 5 & 0 & 3 & 62 & 23 & 17 & 105 & pow $\times \mathrm{d} \log t+\mathrm{b}$ & 5 & 33 & 35 & 24 & 0 & 92 \\
\hline hyp $\times$ hypp $+b$ & 5 & 54 & 29 & 5 & 3 & 16 & 107 & hyp $\times d \log t+b$ & 5 & 34 & 34 & 29 & 9 & 106 \\
\hline hyp $\times \operatorname{expp}+b$ & 5 & 61 & 35 & 4 & 4 & 18 & 122 & $\exp \times d \log t+b$ & 5 & 28 & 35 & 28 & 16 & 107 \\
\hline hyp $\times$ hyp $+b$ & 4 & 77 & 16 & 4 & 14 & 19 & 130 & apow $\times$ hyp $+b$ & 5 & 64 & 36 & 17 & 15 & 132 \\
\hline pow $\times \operatorname{expp}+b$ & 5 & 12 & 18 & 59 & 14 & 28 & 131 & pow $\times$ hyp $+b$ & 4 & 78 & 70 & 7 & 10 & 165 \\
\hline pow $\times$ hyp $+b$ & 4 & 15 & 0 & 57 & 23 & 41 & 136 & $\operatorname{logt} \times$ hyp $+b$ & 5 & 84 & 55 & 19 & 15 & 173 \\
\hline aexp $\times$ hyp $+b$ & 5 & 76 & 30 & 0 & 16 & 24 & 146 & ahyp $\times$ hyp $+b$ & 5 & 99 & 45 & 16 & 22 & 182 \\
\hline pow $\times$ hypp $+b$ & 5 & 11 & 25 & 60 & 29 & 31 & 156 & aexp $\times$ hyp $+b$ & 5 & 102 & 58 & 17 & 19 & 196 \\
\hline ahyp $\times$ hyp $+b$ & 5 & 94 & 35 & 0 & 24 & 27 & 180 & hyp $\times$ hyp $+b$ & 4 & 114 & 78 & 6 & 14 & 212 \\
\hline apow $\times$ hyp $+b$ & 5 & 31 & 19 & 59 & 33 & 50 & 192 & exp $\times$ hyp $+b$ & 4 & 107 & 100 & 8 & 14 & 229 \\
\hline $\log t \times h y p+b$ & 5 & 24 & 118 & 7 & 51 & 7 & 207 & expp $\times h y p+b$ & 5 & 108 & 91 & 14 & 15 & 228 \\
\hline pow $\times$ hyp & 3 & 76 & 80 & 56 & 13 & 206 & 431 & pow $\times$ hyp & 3 & 80 & 44 & 0 & 321 & 445 \\
\hline hyp ×hyp & 3 & 120 & 224 & 15 & 9 & 294 & 662 & hyp ×hyp & 3 & 120 & 64 & 19 & 321 & 524 \\
\hline exp $\times h y p$ & 3 & 122 & 235 & 24 & 3 & 360 & 744 & exp $\times$ hyp & 3 & 141 & 272 & 31 & 318 & 762 \\
\hline Davison \& Hunter $\dagger$ & 3 & 105 & 252 & 13 & 22 & 387 & 779 & Davison \& Hunter $\dagger$ & 3 & 336 & 367 & 118 & 462 & 1283 \\
\hline Davison \& Hunter B & 3 & 104 & 260 & 5 & 14 & 440 & 823 & Davison \& Hunter B & 3 & 350 & 395 & 149 & 477 & 1371 \\
\hline Stevens & 2 & 87 & 245 & 88 & 6 & 470 & 896 & Stevens & 2 & 373 & 384 & 141 & 473 & 1371 \\
\hline Herrnstein & 2 & 156 & 489 & 41 & 30 & 644 & 1360 & Herrnstein & 2 & 453 & 737 & 274 & 679 & 2143 \\
\hline
\end{tabular}




\section{APPENDIX B}

\section{DYNAMICAL MODELING}

Elliffe and Davison's (2010) data represent behavioral dynamics, where behavior was recorded for each reinforcer in a sequence of 10 . In their experiment, a four-way reinforcer ratio across four alternatives changed every 10 reinforcers.

To model this data set, we used the linearization technique described in Navakatikyan and Davison (2010) in detail. Unlike the usual dynamic analysis, where steady states are found from differential equations, this technique allows the use of equations of steady state (which are our LOE models) to analyze dynamical data. Such data are modeled sequentially, using behavior calculated for the present step to predict behavior in the next step, and so on.

In brief, any of the LOE models can be used to describe each next value of dynamical behavior using the current values of behavior and reinforcer rate according to the following equation:

$$
B_{i+1}^{*}=B_{i}^{*}+k_{t}\left[B\left(R_{i}\right)-B_{i}^{*}\right]
$$

where $B^{*}{ }_{i+1}$ and $B^{*}{ }_{i}$ are the next and current value of behavior, respectively; $B\left(R_{\mathrm{i}}\right)$ is behavior in the steady state calculated from one of LOE models under investigation using the current obtained reinforcer rate (i.e. $\left.R_{\mathrm{i}}\right) ; k_{t}$ is a dynamic constant, which is a fraction of $\left(B\left(R_{\mathrm{i}}\right)-B_{\mathrm{i}}{ }^{*}\right)$ that changes each reinforcer and has the dimension of reinforcers ${ }^{-1}$. Equation B1 says that change in behavior is directly proportional to the difference between current and steady-state behavior.

In practice we cannot predict all 10 values of behavior in a sequence of 10 , because for the first step we don't know what the value of previous behavior was. Therefore, we use the observed behavior in step 1 as $B_{1} *$ to calculate the predicted behavior at step 2, i.e. $B_{2}{ }^{*}$, and then the predicted behavior at step 2 to calculate the predicted behavior at step 3, and so on. Thus we have nine predicted values of behavior for steps 2 to 10 . These nine predicted values are used for optimization. 


\section{APPENDIX C}

\section{DATA ANALYSIS}

Statistical analysis was done using the R programming language. Nonlinear least squares optimization was performed using the $n l s$ function from the stats package in R. Log-likelihood value $\left(\log _{\mathrm{e}} L\right)$ for a model was determined using the $\log L i k$ function. For each equation we conducted 20 optimizations, using random combinations of the initial values, and the best were selected to be a final solution. Parameters of the models were bounded. Upper bounds for $B_{\max }, A$ and $B$ were set to 150 , for biases to 30 , and to 300 for other coefficients. Lower bounds were usually set at 0.000001 , but sometimes were increased to ensure convergence.

\section{Goodness of Fit and Model Comparison}

As a goodness of fit measure we used variance accounted for (VAC).

$$
\operatorname{VAC}=100 \times\left\{\left[\operatorname{Var}_{p}(y)-R S S / n\right] / \operatorname{Var}_{p}(y)\right\}
$$

where $\operatorname{Var}_{\mathrm{p}}(y)$ is the population (not sample) variance of behavioral index $y ; R S S$ is residual sum of squares of the fitted model, $y_{\mathrm{i}}$ and $\bar{y}$ are the $i^{\text {th }}$ value and mean of $y$ respectively, and $n$ is number of data points. Unless otherwise stated we present a median value of VAC across subjects' models.

However, as the models have different numbers of adjustable parameters, to select the best model we used a conservative Bayesian information criterion (BIC) and the less conservative second-order (or corrected) Akaike information criterion (AICc). We used the formulae derived for series of data, i.e., for models fitted individually to a series of subjects in an experimental group (McArdle, personal communication, 2005; see Navakatikyan, 2007; Navakatikyan \& Davison, 2010).

In the present article instead of RSS to calculate BIC and AICc we used log-likelihoods:

$$
\sum_{i=1}^{N}\left(n_{i} \cdot \log _{e} \frac{R S S_{i}}{n_{i}}\right)=-2 \sum_{i=1}^{N}\left(\log _{e} L\right) .
$$

$i$ is index of a subject set $1,2, \ldots N$.

The AICc is recommended for theoretical reasons (Burnham \& Anderson, 1998) when the sample size is small (the ratio $n / K$ is less than 40 ). In our analysis the criteria produced very similar results, but AICc returned slightly more consistent rankings of models across data sets. For that reason we will present only the AICc analysis. 
Conventionally, models are compared using the differences ( $\mathrm{AICc}$ ) between values of a model's AICc and the AICc of the best model, as the differences, unlike absolute value, are independent of the dimension of RSS. A cutoff value of 6 for a difference in information criteria is considered to be an evidence for a model to be a better data description. A difference of 10 or more means that there is virtually no support for a model with the larger AICc value being a better description, or that there is strong evidence for another model to be a better data description (Burnham \& Anderson, 1998). However "The reader should not take these guidelines as inviolate since there are situations to which they do not apply well (such as when there is a small sample size or dependent observations). Likewise, if there are thousands of models, these guidelines may not hold." (Burnham \& Anderson, 1998, p. 170). We do not compare thousands of models, but the small sample size and dependent observations are always a possibility in behavioral studies. Thus, as a minimal precaution against spurious conclusion we chose the largest cutoff value of 10 .

To aggregate the results over all experiments the models were ordered according to the sums of $\triangle \mathrm{AICc}$ over the experiments. This procedure can be justified because a single model fitting all the experiments at once (using experiment as a factor) would, since the experiments are independent, have a $\log$ likelihood equal to the sum of the individual log likelihoods. The models can therefore be ranked using the sum of the $\triangle \mathrm{AICc}$ (Brian McArdle, personal communication). Alternatively, the sum of the models' ranks over the experiments could have been used.

\section{Transformations}

Three types of transformations were applied to behavioral indices to ensure the best homogeneity of variance and normality of data distribution, $\log (y), \log (y+1)$ and no transformation. AICc values reported are related to the models fitted to the transformed values. VACs are reported for transformed values only, as VAC for nontransformed values were very similar. The transformation is a usual approach to ensure that the statistical conclusions, in particular, values of AICc are valid.

\section{Diagnostics}

For each model (called in this section main models) we assessed the presence of trend, normality of distribution and equality of variance of the conditional residuals. Trend. The presence of trend was assessed by constructing a linear model of residuals vs. main model fitted values. The linear, quadratic and cubic models were fitted sequentially using the $\operatorname{lm}$ function in $\mathrm{R}$, and the highest-power significant model was analyzed. If none of the models were significant, i.e., the null hypothesis that all coefficients equal 0 was true, the test for the absence of trend was considered passed. In any case, the $p$-value and the value of $R^{2}$ were calculated to estimate the degree of 
violation of the assumption of no trend. Normality. Normality of the residual distribution was assessed using a Shapiro-Wilks test. Equality of variance. The equality of variance was assessed by constructing a linear model of squared residuals vs. main model fitted values. The analysis was conducted in the same way as for the analysis of trend, but the upper polynomial was quadratic. In general, the best models passed the diagnostic tests adequately, and the exceptions are mentioned below. 


\section{APPENDIX D}

\section{Median Values of Parameters for The Best Presented Models}

Table D1

Parameters of the best models. Median values are presented, except for Davison and Hunter's (1976) experiment where modeling was performed on data averaged over pigeons. B, ResT and $\mathrm{CO}$ are response rate, residence time and changeover rate; $\log$ is logarithmic transformation. $B_{\max }$ and $A$ are maximum overall behavior or scaling parameter; $k$ and $d$ parameters control the rate of increase in behavior with increase in reinforcer rate on the present alternative; $k_{\text {red }}$ and $s$ parameters control the rate of decrease in behavior with increase in reinforcer rate on the other alternatives; $B_{\mathrm{a}}$ is a baseline constant, $k_{\mathrm{t}}$ is a time constant. Abbreviations of the models are as in Table 1 .

\begin{tabular}{|c|c|c|c|c|c|c|c|c|}
\hline \multirow[b]{2}{*}{ Data } & \multirow[b]{2}{*}{$\begin{array}{l}\text { Behavioral } \\
\text { index }\end{array}$} & \multicolumn{7}{|c|}{ Parameters } \\
\hline & & $\begin{array}{l}\text { Model } \\
\text { description }\end{array}$ & $\begin{array}{l}B_{\max }, \\
\text { or } A\end{array}$ & $\begin{array}{l}k, \text { or } \\
d\end{array}$ & $k_{\text {red }}$ & $S$ & $B_{\mathrm{a}}$ & $k_{\mathrm{t}}$ \\
\hline \multirow{2}{*}{$\begin{array}{l}\text { Davison \& McCarthy } \\
\text { (1994) }\end{array}$} & $\log \mathrm{B}$ & pow $\times$ hypp $+b$ & 150.0 & 1.40 & 0.06 & 0.70 & 4.06 & - \\
\hline & $\log$ ResT & pow $\times$ hypp $+b$ & 65.2 & 0.16 & 0.28 & 1.95 & 2.4 & - \\
\hline \multirow{2}{*}{ Murrell (1976) } & $\log \mathrm{B}$ & pow $\times$ hyp + b & 45.1 & 0.85 & 0.53 & - & 1.2 & - \\
\hline & $\log \operatorname{ResT}$ & hyp $\times$ hypp $+b$ & 88.0 & 0.0002 & 0.26 & 1.70 & 2.4 & - \\
\hline$r(1976)$ & $\log (B+1)$ & hyp $\times$ hyp + b & 106.4 & 0.22 & 1.41 & - & 0.4 & - \\
\hline \multirow{2}{*}{ Pliskoff \& Brown (1976) } & $\log (\mathrm{B}+1)$ & hyp $\times$ hypp $+b$ & 95.6 & 0.11 & 0.57 & 3.45 & 0.1 & - \\
\hline & $\log \operatorname{ResT}$ & pow $\times$ hyp & 77.8 & 0.24 & 0.03 & - & - & - \\
\hline \multirow{3}{*}{ Elliffe \& Davison (2010) } & $\mathrm{B}$ & hyp ×hypp + b & 150.0 & 0.93 & 0.77 & 10.4 & 1.15 & 0.24 \\
\hline & $\mathrm{CO}$ & hyp $\times$ hypp + b & 78.8 & 0.66 & 0.32 & 1.3 & 0.89 & 0.23 \\
\hline & ResT & pow $\times$ hyp + b & 8.6 & 0.37 & 0.19 & - & 4.8 & 0.22 \\
\hline
\end{tabular}




\section{APPENDIX E}

\section{DATA From MurReLL'S (1995) EXPERIMENT, PART B}

Table E1

Sequence of experimental conditions, number of sessions in each condition, overall probability of reinforcement per second, and arranged relative reinforcer frequency for each alternative in Murrell's (1995) experiment, Part B. $\mathrm{R}, \mathrm{G}$ and $\mathrm{Y}$ are the red, green and yellow alternatives, denoted as alternatives 1,2 and 3. Probabilities of the main key colors are all $1 / 3$. Blackout $=3 \mathrm{~s}$.

\begin{tabular}{|c|c|c|c|c|c|}
\hline \multirow{2}{*}{ Conditions } & \multicolumn{4}{|c|}{ Reinforcer probability per second } & \multirow{2}{*}{ Sessions } \\
\cline { 2 - 5 } & $R$ & $G$ & $Y$ & Overall & \\
\hline 7 & 0.0067 & 0.0011 & 0.0144 & 0.0222 & 45 \\
8 & 0.0022 & 0.0133 & 0.0067 & 0.0222 & 32 \\
9 & 0.0100 & 0.0017 & 0.0105 & 0.0222 & 50 \\
10 & 0.0028 & 0.0167 & 0.0028 & 0.0223 & 32 \\
11 & 0.0133 & 0.0022 & 0.0067 & 0.0222 & 42 \\
12 & 0.0011 & 0.0067 & 0.0144 & 0.0222 & 42 \\
13 & 0.0167 & 0.0028 & 0.0028 & 0.0223 & 22 \\
14 & 0.0017 & 0.0100 & 0.0105 & 0.0222 & 29 \\
15 & 0.0190 & 0.0032 & 0 & 0.0222 & 24 \\
16 & 0.0032 & 0.0190 & 0 & 0.0222 & 29 \\
\hline
\end{tabular}


Table E2

Number on responses emitted, seconds spent responding (excluding blackout periods after changeovers), reinforcers obtained on each alternative and number of changeovers from an alternative to two other alternatives that were used to calculate the residence time. All values are sums over the last six sessions of the condition. R, G and $\mathrm{Y}$ are red, green and yellow alternative color of the main key, denoted as alternatives 1,2 and 3.

\begin{tabular}{|c|c|c|c|c|c|c|c|c|c|c|c|c|c|}
\hline \multirow[b]{2}{*}{ Bird } & \multirow[b]{2}{*}{ Condition } & \multicolumn{3}{|c|}{ Responses } & \multicolumn{3}{|c|}{ Time $(s)$} & \multicolumn{3}{|c|}{ Changeovers } & \multicolumn{3}{|c|}{ Reinforcers } \\
\hline & & $R$ & $G$ & $Y$ & $R$ & $G$ & $Y$ & $R$ & $G$ & $Y$ & $R$ & $G$ & $Y$ \\
\hline \multirow[t]{10}{*}{41} & 7 & 1323 & 489 & 2791 & 2628 & 2069 & 3331 & 475 & 456 & 374 & 59 & 14 & 162 \\
\hline & 8 & 595 & 2648 & 1294 & 1839 & 3539 & 2412 & 500 & 401 & 468 & 22 & 153 & 63 \\
\hline & 9 & 2241 & 507 & 1826 & 3105 & 2024 & 2261 & 391 & 515 & 431 & 119 & 11 & 108 \\
\hline & 10 & 689 & 4122 & 845 & 1669 & 4256 & 1743 & 443 & 339 & 437 & 39 & 168 & 26 \\
\hline & 11 & 2136 & 755 & 1340 & 3158 & 1859 & 2295 & 366 & 418 & 370 & 121 & 33 & 78 \\
\hline & 12 & 505 & 1185 & 3686 & 1787 & 2144 & 3862 & 462 & 407 & 398 & 13 & 81 & 141 \\
\hline & 13 & 5863 & 554 & 885 & 6049 & 1357 & 1757 & 220 & 335 & 334 & 178 & 26 & 31 \\
\hline & 14 & 652 & 2113 & 2289 & 1946 & 2984 & 3064 & 496 & 434 & 421 & 11 & 106 & 112 \\
\hline & 15 & 6949 & 442 & 167 & 7192 & 1403 & 1445 & 149 & 283 & 322 & 208 & 24 & 0 \\
\hline & 16 & 963 & 6744 & 152 & 1856 & 6273 & 1453 & 384 & 243 & 405 & 42 & 193 & 0 \\
\hline \multirow{10}{*}{42} & 7 & 1051 & 684 & 5329 & 1729 & 1653 & 6751 & 273 & 310 & 227 & 77 & 10 & 142 \\
\hline & 8 & 901 & 3215 & 1795 & 2025 & 4211 & 2990 & 379 & 277 & 355 & 19 & 144 & 69 \\
\hline & 9 & 3588 & 857 & 2888 & 3867 & 1656 & 3155 & 357 & 415 & 357 & 107 & 15 & 118 \\
\hline & 10 & 910 & 4610 & 1052 & 1823 & 5597 & 2496 & 335 & 271 & 353 & 26 & 161 & 30 \\
\hline & 11 & 3376 & 889 & 1349 & 4080 & 1422 & 2018 & 342 & 405 & 399 & 131 & 24 & 84 \\
\hline & 12 & 584 & 1567 & 3044 & 2246 & 2671 & 3830 & 379 & 325 & 298 & 14 & 71 & 148 \\
\hline & 13 & 5419 & 836 & 1044 & 6063 & 1596 & 2060 & 276 & 360 & 374 & 173 & 30 & 23 \\
\hline & 14 & 943 & 2671 & 3113 & 1878 & 3014 & 3518 & 373 & 318 & 313 & 18 & 107 & 113 \\
\hline & 15 & 6831 & 680 & 427 & 7016 & 1224 & 1360 & 169 & 296 & 301 & 208 & 32 & 0 \\
\hline & 16 & 1157 & 6489 & 436 & 1868 & 6812 & 1274 & 314 & 174 & 318 & 33 & 207 & 0 \\
\hline \multirow[t]{10}{*}{43} & 7 & 993 & 263 & 4591 & 2435 & 2331 & 7699 & 192 & 218 & 150 & 52 & 8 & 124 \\
\hline & 8 & 571 & 3070 & 1299 & 2276 & 5620 & 3702 & 260 & 190 & 219 & 16 & 121 & 64 \\
\hline & 9 & 1644 & 525 & 1950 & 4158 & 4217 & 3709 & 193 & 225 & 191 & 75 & 19 & 95 \\
\hline & 10 & 689 & 5089 & 703 & 2703 & 6017 & 2732 & 275 & 166 & 260 & 19 & 168 & 22 \\
\hline & 11 & 3491 & 621 & 1131 & 4569 & 3233 & 3440 & 221 & 277 & 255 & 131 & 22 & 63 \\
\hline & 12 & 763 & 1130 & 2556 & 3076 & 4524 & 4652 & 238 & 208 & 164 & 15 & 59 & 127 \\
\hline & 13 & 4063 & 703 & 1389 & 4365 & 4361 & 2842 & 164 & 264 & 257 & 150 & 30 & 27 \\
\hline & 14 & 658 & 2666 & 2467 & 2738 & 4285 & 4279 & 293 & 238 & 219 & 19 & 92 & 104 \\
\hline & 15 & 6572 & 528 & 251 & 6760 & 2524 & 2460 & 93 & 206 & 221 & 200 & 27 & 0 \\
\hline & 16 & 595 & 5194 & 162 & 2383 & 6555 & 1921 & 210 & 106 & 230 & 31 & 202 & 0 \\
\hline \multirow[t]{10}{*}{44} & 7 & 1678 & 410 & 3678 & 2738 & 1874 & 5349 & 325 & 363 & 266 & 63 & 10 & 153 \\
\hline & 8 & 870 & 3694 & 1528 & 2272 & 4372 & 2708 & 413 & 302 & 361 & 20 & 140 & 68 \\
\hline & 9 & 2360 & 824 & 2356 & 3899 & 2555 & 3931 & 291 & 344 & 259 & 92 & 16 & 113 \\
\hline & 10 & 662 & 6417 & 725 & 1858 & 7358 & 1984 & 264 & 165 & 270 & 29 & 171 & 21 \\
\hline & 11 & 4089 & 1079 & 1341 & 5148 & 2602 & 2891 & 261 & 334 & 296 & 134 & 26 & 64 \\
\hline & 12 & 577 & 1651 & 2701 & 2468 & 3344 & 4719 & 329 & 320 & 262 & 16 & 61 & 143 \\
\hline & 13 & 6257 & 772 & 967 & 7582 & 1706 & 2068 & 179 & 277 & 277 & 172 & 27 & 27 \\
\hline & 14 & 596 & 2394 & 2576 & 2271 & 4044 & 4242 & 345 & 307 & 285 & 17 & 90 & 103 \\
\hline & 15 & 6865 & 777 & 363 & 7363 & 1970 & 1753 & 132 & 251 & 264 & 202 & 29 & 0 \\
\hline & 16 & 548 & 6403 & 260 & 1502 & 7979 & 1713 & 258 & 166 & 284 & 30 & 200 & 0 \\
\hline \multirow[t]{3}{*}{45} & 7 & 1673 & 575 & 5754 & 2104 & 1980 & 5024 & 347 & 394 & 318 & 68 & 11 & 147 \\
\hline & 8 & 730 & 5737 & 2180 & 2149 & 4821 & 2553 & 366 & 257 & 333 & 22 & 149 & 69 \\
\hline & 9 & 2942 & 853 & 4481 & 3228 & 2595 & 4354 & 290 & 349 & 289 & 106 & 9 & 111 \\
\hline
\end{tabular}




\begin{tabular}{|r|r|rrr|rrr|rrr|rrr|}
\hline \multirow{6}{*}{10} & 786 & 7545 & 663 & 1915 & 7691 & 2094 & 230 & 148 & 238 & 32 & 152 & 29 \\
& 11 & 5138 & 990 & 1376 & 4682 & 2774 & 2621 & 234 & 349 & 301 & 148 & 24 & 62 \\
& 12 & 752 & 1402 & 5249 & 2657 & 2615 & 5259 & 325 & 284 & 233 & 8 & 78 & 141 \\
& 13 & 7030 & 606 & 810 & 6594 & 2533 & 2406 & 137 & 224 & 235 & 162 & 23 & 27 \\
& 14 & 635 & 2255 & 3492 & 2627 & 3204 & 3736 & 395 & 335 & 321 & 17 & 119 & 104 \\
& 15 & 9649 & 485 & 218 & 8153 & 1478 & 1563 & 105 & 214 & 258 & 207 & 26 & 0 \\
& 16 & 1248 & 9520 & 134 & 2432 & 8298 & 1460 & 204 & 87 & 225 & 40 & 196 & 0 \\
\hline 46 & 7 & 535 & 216 & 5096 & 1988 & 1739 & 6755 & 325 & 383 & 248 & 53 & 7 & 148 \\
& 8 & 613 & 3240 & 1006 & 2068 & 4391 & 2651 & 447 & 335 & 397 & 18 & 135 & 59 \\
& 9 & 1530 & 558 & 2073 & 2694 & 2015 & 3547 & 363 & 409 & 363 & 95 & 28 & 116 \\
& 10 & 522 & 6991 & 538 & 1379 & 6914 & 1300 & 300 & 222 & 276 & 29 & 177 & 30 \\
& 11 & 2275 & 936 & 1406 & 3523 & 2413 & 2499 & 436 & 477 & 471 & 122 & 23 & 57 \\
& 12 & 553 & 1685 & 4183 & 2442 & 2722 & 4172 & 403 & 352 & 320 & 7 & 74 & 137 \\
& 13 & 8253 & 694 & 715 & 6276 & 1938 & 2208 & 220 & 298 & 310 & 164 & 32 & 29 \\
& 14 & 440 & 1396 & 2023 & 1970 & 2634 & 3032 & 454 & 406 & 400 & 12 & 105 & 120 \\
& 15 & 8843 & 507 & 202 & 6369 & 1338 & 1480 & 207 & 307 & 344 & 204 & 35 & 0 \\
& 16 & 509 & 8591 & 184 & 1510 & 6505 & 1359 & 304 & 196 & 337 & 28 & 211 & 0 \\
\hline
\end{tabular}




\section{APPENDIX D}

\section{MEDIAN VALUES OF PARAMETERS FOR THE BEST MODELS}

Table D1

Parameters of the best models. Median values are presented, except for Davison and Hunter's (1976) experiment where modeling was performed on data averaged over pigeons. B, ResT and $\mathrm{CO}$ are response rate, residence time and changeover rate; $\log$ is logarithmic transformation. $B_{\max }$ and $A$ are maximum overall behavior or scaling parameter; $k$ and $d$ parameters control the rate of increase in behavior with increase in reinforcer rate on the present alternative; $k_{\text {red }}$ and $s$ parameters control the rate of decrease in behavior with increase in reinforcer rate on the other alternatives; $B_{\mathrm{a}}$ is a baseline constant, $k_{\mathrm{t}}$ is a time constant. Abbreviations of the models are as in Table 1 .

\begin{tabular}{|c|c|c|c|c|c|c|c|c|}
\hline \multirow[b]{2}{*}{ Data } & \multirow[b]{2}{*}{$\begin{array}{l}\text { Behavioral } \\
\text { index }\end{array}$} & \multicolumn{7}{|c|}{ Parameters } \\
\hline & & $\begin{array}{l}\text { Model } \\
\text { description }\end{array}$ & $\begin{array}{l}B_{\max }, \\
\text { or } A\end{array}$ & $\begin{array}{l}k, \text { or } \\
d\end{array}$ & $k_{\text {red }}$ & $s$ & $B_{\mathrm{a}}$ & $k_{\mathrm{t}}$ \\
\hline \multirow{2}{*}{$\begin{array}{l}\text { Davison \& McCarthy } \\
\text { (1994) }\end{array}$} & $\log B$ & pow $\times$ hypp $+b$ & 150.0 & 1.40 & 0.06 & 0.70 & 4.06 & \\
\hline & $\log \operatorname{ResT}$ & pow $\times$ hypp $+b$ & 65.2 & 0.16 & 0.28 & 1.95 & 2.4 & - \\
\hline \multirow{2}{*}{ Murrell (1976) } & $\log B$ & pow $\times$ hyp + b & 45.1 & 0.85 & 0.53 & - & 1.2 & - \\
\hline & $\log$ ResT & hyp $\times$ hypp $+b$ & 88.0 & 0.0002 & 0.26 & 1.70 & 2.4 & - \\
\hline Hunter (1976) & $\log (B+1)$ & hyp $\times$ hyp + b & 106.4 & 0.22 & 1.41 & - & 0.4 & - \\
\hline \multirow{2}{*}{ Pliskoff \& Brown (1976) } & $\log (B+1)$ & hyp $\times$ hypp $+b$ & 95.6 & 0.11 & 0.57 & 3.45 & 0.1 & - \\
\hline & $\log \operatorname{ResT}$ & pow $\times$ hyp $+b$ & 79.9 & 0.36 & 0.03 & - & 0.4 & - \\
\hline \multirow{3}{*}{ Elliffe \& Davison (2010) } & $\mathrm{B}$ & hyp xhypp + b & 150.0 & 0.93 & 0.77 & 10.4 & 1.15 & 0.24 \\
\hline & $\mathrm{CO}$ & hyp $\times$ hypp + b & 78.8 & 0.66 & 0.32 & 1.3 & 0.89 & 0.23 \\
\hline & ResT & pow $\times$ hyp $+b$ & 8.6 & 0.37 & 0.19 & - & 4.8 & 0.22 \\
\hline
\end{tabular}

